\title{
Article \\ An Investigation on the Vortex Effect of a CALM Buoy under Water Waves Using Computational Fluid Dynamics (CFD)
}

\author{
Chiemela Victor Amaechi ${ }^{1,2, *(D)}$ and Jianqiao $\mathrm{Ye}^{1, *}$
}

1 Department of Engineering, Lancaster University, Lancaster LA1 4YR, Lancashire, UK

2 Standard Organisation of Nigeria (SON), 52 Lome Crescent, Wuse Zone 7, Abuja 900287, Nigeria

* Correspondence: c.amaechi@lancaster.ac.uk or chiemelavic@gmail.com (C.V.A.); j.ye2@lancaster.ac.uk (J.Y.)

Citation: Amaechi, C.V.; Ye, J. An Investigation on the Vortex Effect of a CALM Buoy under Water Waves Using Computational Fluid

Dynamics (CFD). Inventions 2022, 7

23. https://doi.org/10.3390/

inventions7010023

Academic Editors: M. M. Bhatti and Sara I. Abdelsalam

Received: 2 January 2022

Accepted: 1 February 2022

Published: 4 February 2022

Publisher's Note: MDPI stays neutral with regard to jurisdictional claims in published maps and institutional affiliations.

Copyright: (C) 2022 by the authors. Licensee MDPI, Basel, Switzerland. This article is an open access article distributed under the terms and conditions of the Creative Commons Attribution (CC BY) license (https:// creativecommons.org/licenses/by/ $4.0 /)$.

\begin{abstract}
Floating offshore structures (FOS) must be designed to be stable, to float, and to be able to support other structures for which they were designed. These FOS are needed for different transfer operations in oil terminals. However, water waves affect the motion response of floating buoys. Under normal sea states, the free-floating buoy presents stable periodic responses. However, when moored, they are kept in position. Mooring configurations used to moor buoys in single point mooring (SPM) terminals could require systems such as Catenary Anchor Leg Moorings (CALM) and Single Anchor Leg Moorings (SALM). The CALM buoys are one of the most commonly-utilised type of offshore loading terminal. Due to the wider application of CALM buoy systems, it is necessary to investigate the fluid structure interaction (FSI) and vortex effect on the buoy. In this study, a numerical investigation is presented on a CALM buoy model conducted using Computational Fluid Dynamics (CFD) in ANSYS Fluent version R2 2020. Some hydrodynamic definitions and governing equations were presented to introduce the model. The results presented visualize and evaluate specific motion characteristics of the CALM buoy with emphasis on the vortex effect. The results of the CFD study present a better understanding of the hydrodynamic parameters, reaction characteristics and fluid-structure interaction under random waves.
\end{abstract}

Keywords: catenary anchor leg mooring (CALM) buoy; computational fluid dynamics (CFD), numerical modelling; vortex; vortex-induced motion (VIM); fluid-structure interaction (FSI); buoy

\section{Introduction}

In recent times, the most commonly-utilised type of offshore loading terminal is the Catenary Anchor-Leg Mooring (CALM). The CALM buoy is a floating buoy with catenary chain legs secured to anchors or piles that anchor it to the bottom, and the buoy also has attached marine hoses [1-5]. As a result of sheer limited inertia of the CALM buoys, mooring line reactions are extremely sensitive to waves, posing a significant wear risk to the mooring lines. Extreme waves can even cause mooring lines to break and affect the behaviour of marine hoses as reported in some CALM buoy system failures [6-9]. As a result, studying the motions of the CALM buoy in mild, squall and severe wave conditions is extremely important [10-13], as they also influence hose mechanics [14-19]. Over the years, there has been a number of motion response phenomena of floating CALM buoys, however, there are limited computational fluid dynamics (CFD) investigations presented. Different questions have been answered on marine riser mechanics [20-22], CALM buoy dynamics [23-27] and CALM buoy motion stability [28-31], but few works addressed other issues that encompass the motion response of CALM buoy in CFD, moored aspects of single point mooring (SPM) systems, flow vorticity, pressure distribution on CALM buoy, velocity impact on CALM buoy, and vortex-induced motion (VIM) on CALM buoys or similar floating offshore structures (FOS). The sketch the (un)loading operation on a CALM buoy with wave forces and boundary conditions and configured as (a) Chinese-lantern and (b) Lazy-S configurations in Figure 1. It shows the hose-string was attached at an end 
(named 'End A') under the buoy while the second end (named 'End B') connected unto the Pipeline End Manifold (PLEM).
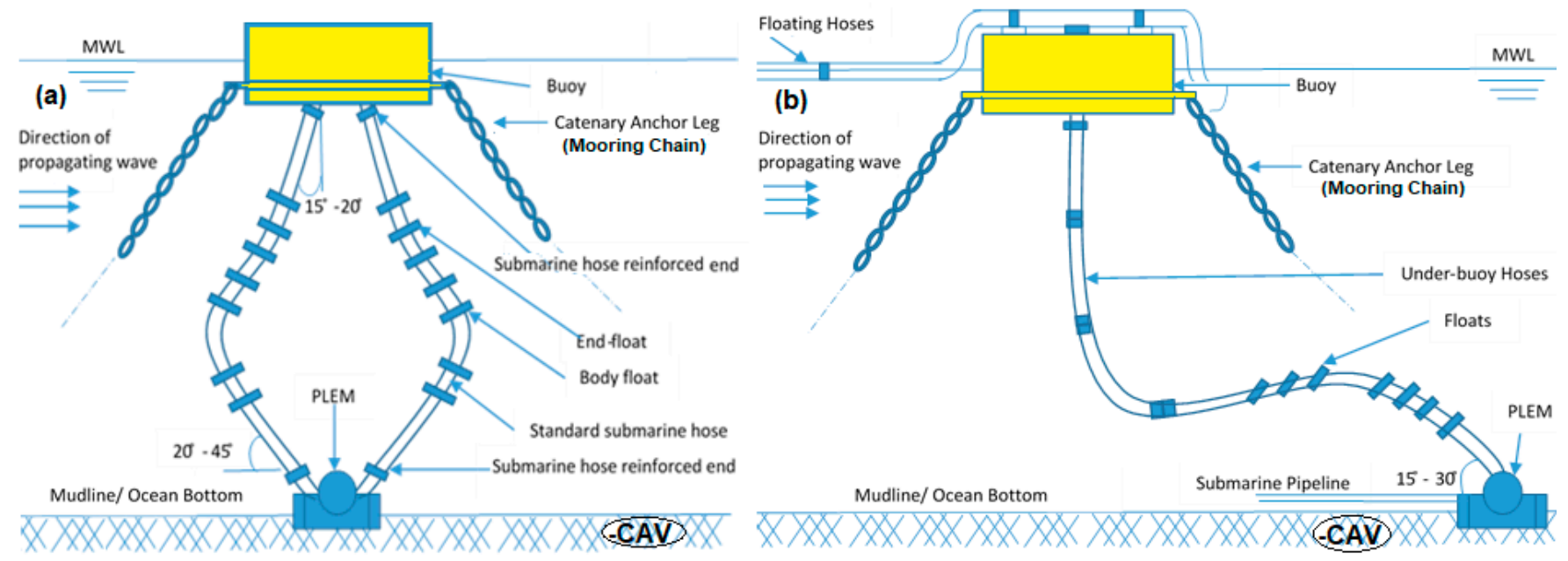

Figure 1. Sketch of loading and offloading operation on a CALM buoy with wave forces and boundary conditions, showing the (a) Chinese-lantern and (b) Lazy-S configurations.

In some recent studies [32-35], coupled simulations of a CALM buoy using a CFDFEM model for wave-induced motion (WIM), whereby the mooring system's FEM model is linked to the CALM buoy CFD model, and the Level-set method is utilised to simulate waves and free surface effects. The authors made use of MOORING3D in conjunction with a motion solver with 6 degrees of freedom (6DoFs). The CFD module calculated the hydrodynamic loading on the moored buoy, using the large eddy simulation (LES) applied on the turbulence model with the Finite-Analytic Navier-Stokes (FANS) code. They concluded that the WIM of the buoy is dominated by inertial and viscous effects of the hull, and that the results of coupled study on free-decay and wave-induced motions match well with model testing. In a similar study, Toxopeus et al. [36] conducted some CFD simulations under waves and calm water using a self-propelled free running 5415M ship model and presented some motion response distributions. Bandringa et al. [37] presented a CFD investigation which was validated using a linked CFD—dynamic mooring model for simulating the behaviour of a shallow water CALM buoy in extreme waves. In their study, a Navier-Stokes based finite-volume, VoF (volume of fluid) CFD solver was coupled with a dynamic mooring model to simulate an interactively moving CALM buoy in a horizontal mooring system. The CFD results were compared to model tests conducted during a ComFLOW-2 joint industry project (JIP) in MARIN's shallow-water basin, and the authors concluded that the validation study focuses on accurately predicting the CALM buoy's coupled responses in extreme, regular shallow-water waves. In addition, they opined that the CFD simulations in which the mooring system is represented by a linearly equivalent spring matrix, including cross terms, are offered as an alternative to simulations with a fully connected dynamic mooring setup. In another JIP called EXPRO-CFD EU FP5 project reported by Woodburn et al. [38,39], some predictions were conducting by utilizing results from a commercial CFD software with existing hydromechanics tools to forecast the response of floating structures in waves and currents, including viscous effects for the response of CALM buoys in waves. The dynamics of the floating structure, its moorings, and risers are modelled using the AQWA-NAUT platform, and CFD delivers the whole set of hydrodynamic forces and moments at each time step in the simulated motion. The CALM buoy has a $23 \mathrm{~m}$ diameter and a $2 \mathrm{~m}$ broad skirt attached $1 \mathrm{~m}$ above the keel; the effects of flow separation off this skirt and the related viscous damping on the buoy's motions were predicted to be considerable, especially around its natural period. Further experiments showed that the flaw in the potential flow technique appeared within the formulated extra viscous damping rather than the drag coefficient model values. Bunnik et al. [40] 
covered experimental work conducted to obtain insight into the tension variations in the mooring lines and export risers of a CALM buoy through a series of model studies. The tests were conducted on a model with on-linearities in the wave forces on the buoy, such as those caused by the presence of the skirt, were investigated via captive experiments in regular and irregular waves. The authors opined that to establish the dampening of the buoy's oscillations and acquire the natural periods, decay tests were necessary as well as the mooring system's dynamics, and the consequent dampening which has substantial impact on the buoy's motions. Different CFD studies include validation studies on CALM buoys presented by various researchers [41-43], application using different CFD modelling methods for fluid studies [44-46] and coupled models [46-48]. Figure 2 shows a typical CALM buoy in the Baltic Sea offshore Lithuania installed by SOFEC [49].

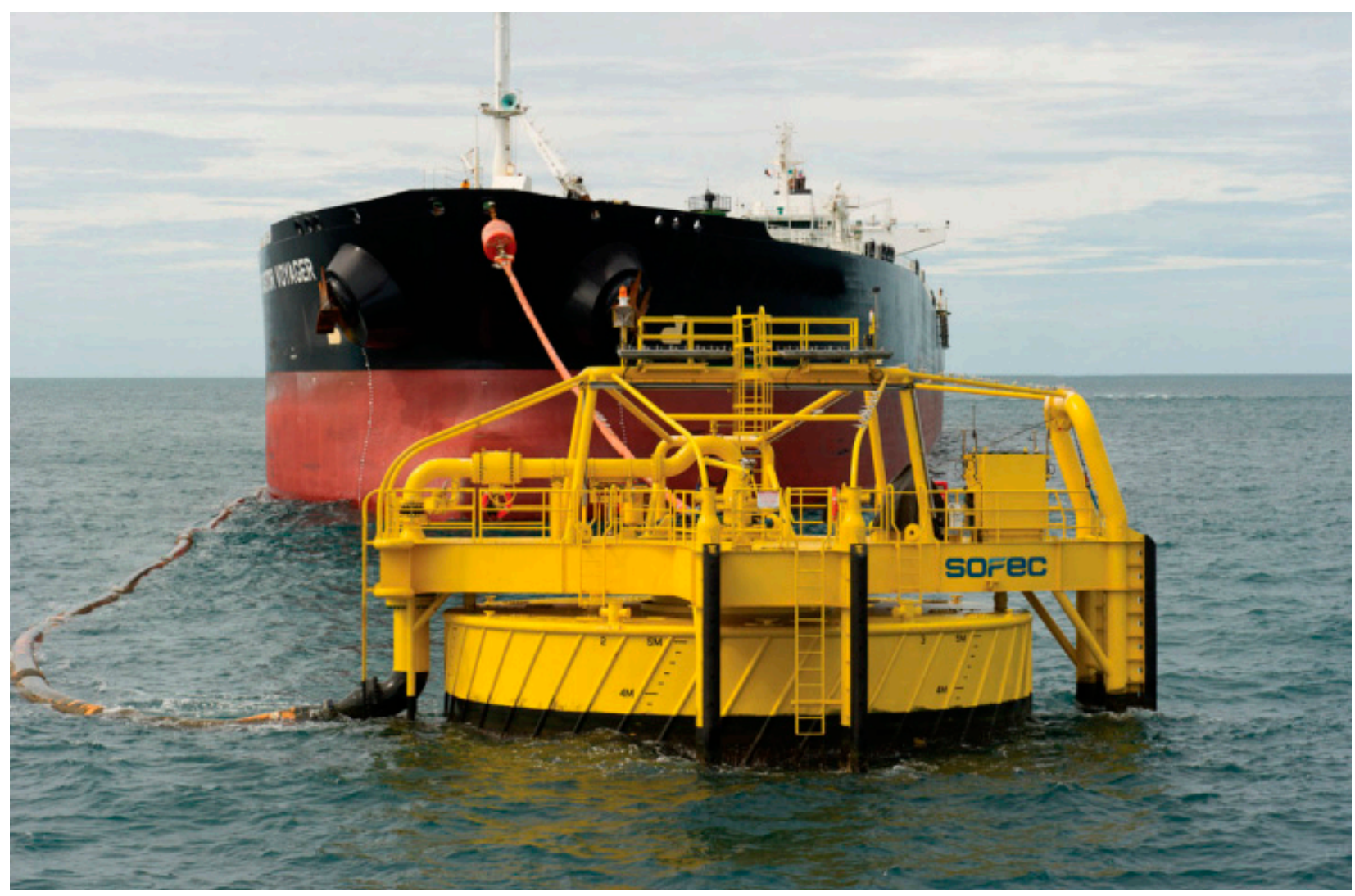

Figure 2. CALM Buoy attached to an FSO in the Baltic Sea offshore Lithuania (Adapted with permission of SOFEC Inc.; Courtesy: SOFEC; Source: [49]).

In this study, a numerical investigation is presented on a CALM buoy model conducted using CFD in ANSYS Fluent version R2 2020. The aim of the research is to investigate the vortex effect of water waves on the CALM buoy. Section 1 presents some background on the research while Section 2 presents some governing equations and theoretical models. Section 3 presents the numerical model for the CFD study while Section 4 presents the results with some discussion. Section 5 presents the concluding remarks on the study.

\section{Theoretical Model}

The theories on the hydrodynamics and statics for CALM buoy with attached hoses is presented in this section.

\subsection{Motion Forces, Drag and Damping Formulation}

The formulation for the drag and damping of the buoy is based on some assumptions. 


\subsubsection{Buoy Model Assumptions}

To achieve this, the following model assumptions are considered in this study:

1. The body of the CALM buoy model is cylindrically shaped;

2. The buoy has a circular skirt attached to it;

3. The skirt is made from solid plates with thin thickness;

4. The skirt is devoid of perforations, except where fairleads or mooring lines are attached;

5. Viscous contributions of damping from skin friction can be neglected;

6. It is assumed that the linear radiation-diffraction computations can be utilised to obtain the CALM buoy's damping and added masses in the following: linear heave, linear surge, and linear pitch;

7. It is assumed that the drag loads on the CALM buoy's bilges are very small;

8. It is assumed that the drag loads on the CALM buoy's skirt can influence the quadratic pitch and heave damping contributions;

9. The local fluid velocity around the skirt's circumferential area utilised in computing these damping contributions. This is conducted by considering the CALM buoys' velocity, but ignoring the flow's disturbance due to the buoy's presence and the wave orbital motions;

10. It is assumed that the CALM buoy hull is positioned in $X-Z$ axes, and subject to a flow direction;

11. The buoy has 6 degrees of freedom (6DoFs) as illustrated in Figure 3. The buoy is considered typically as a single system, and as a floating buoy with a rigid body.

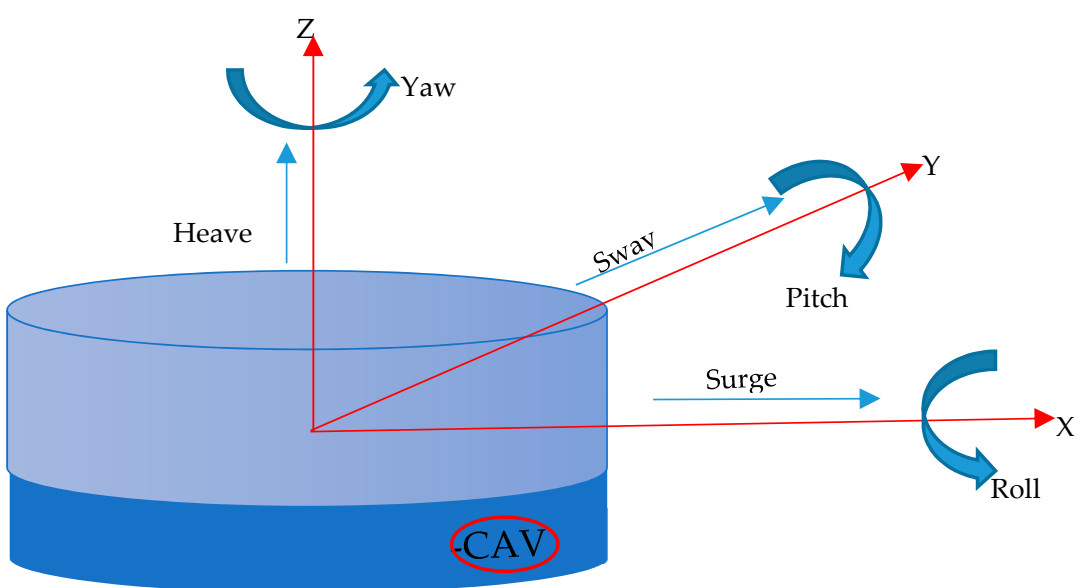

Figure 3. The $6 \mathrm{DoF}$ of a floating CALM buoy, showing the heave, yaw, sway, pitch, surge and roll motions.

\subsubsection{Added Mass \& Damping Coefficients}

The experimental investigation by Cozijn et al. [50,51] were conducted using forced oscillations for heave and pitch damping on CALM buoy. In that study, the loads in the 6-component force frame, as well as the motions of the CALM buoy model, were measured. The measured signals were subjected to a harmonic analysis, where the applied motion is used as the lead signal. The very first harmonic is the observed loads' amplitudes and phase angles. The damping coefficient and the added mass coefficient were calculated using the CALM buoy's motion. Figure 4 is the coordinate system of the CALM buoy hull.

Cozijn et al. [51] obtained the expressions for typical heave motion as given in Equations (1) and (2), where $M$ denotes the dry mass of the CALM buoy model, $C_{z z}$ denotes the heave hydrostatic restoring force coefficient, $\varepsilon F_{z}$ and $F_{z}$ denote the measured heave force amplitude, and $\omega$ denotes the amplitude and frequency of the applied heave motion, and phase lag.

$$
A_{z z}(\omega)=\frac{F_{z} \cdot \cos \left(\varepsilon F_{z}\right)-C_{z z} \cdot z_{a}}{-\omega \cdot z_{a}}-M
$$




$$
B_{z z}(\omega)=\frac{-F_{z} \cdot \sin \left(\varepsilon F_{z}\right)}{-\omega \cdot z_{a}}
$$

(a) Side view of CALM Buoy

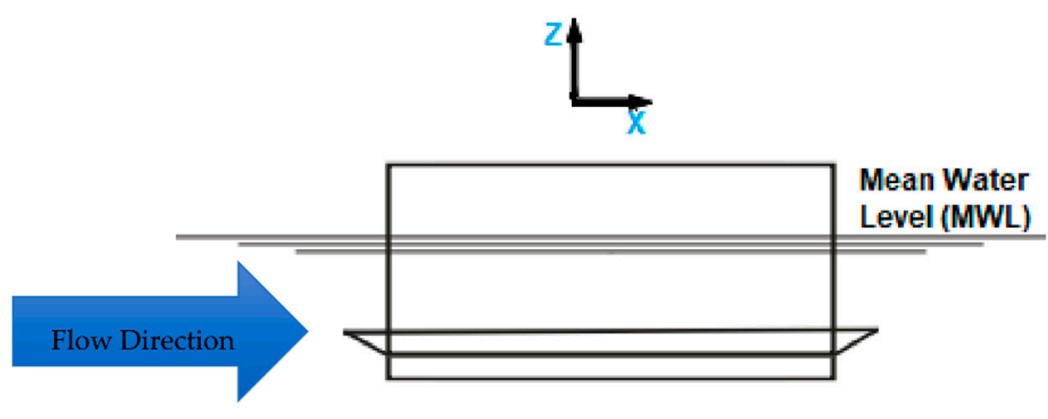

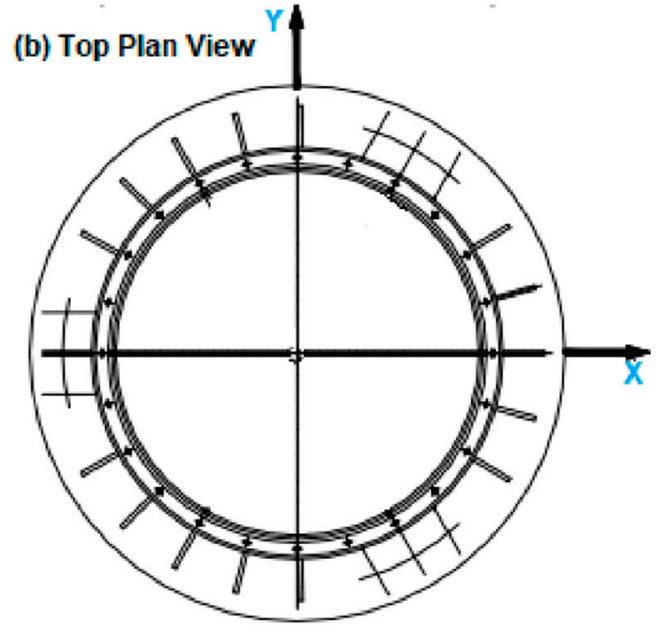

Figure 4. Definition sketch for the Coordinate System of CALM buoy Model (not drawn to scale).

\subsubsection{Load Computations on Buoy's Skirt}

The computation for the loads on the skirt are based on the CALM buoy's geometry, as illustrated in Figure 5. It shows the diameter of the skirt, $D_{S}$, the diameter of the CALM buoy's body, $D_{B}$, the tangential angle obtained from the skirt's circumference, $\alpha$.

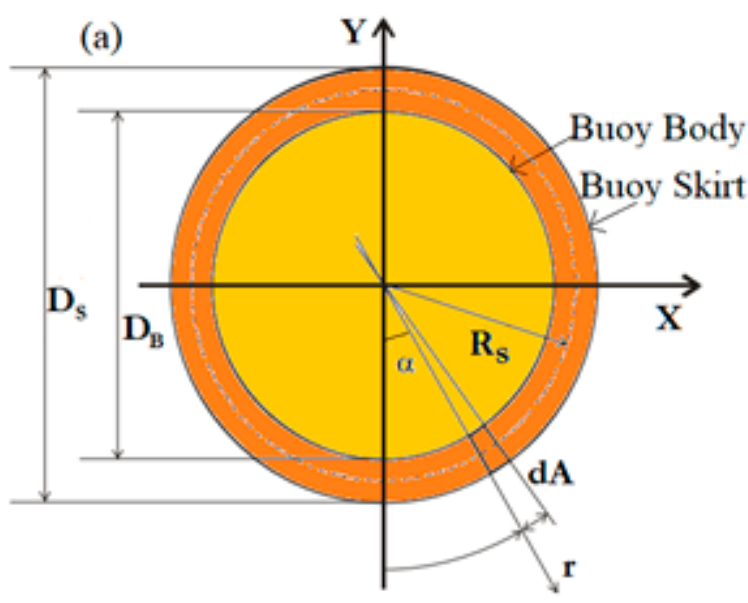

(b)

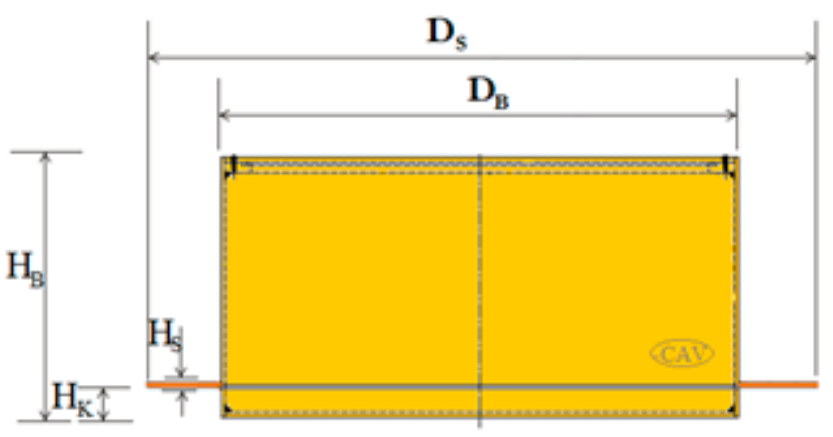

Figure 5. CALM Buoy Model with Skirt Dimension, showing (a) top plan view and (b) side view.

Based on the assumption that the buoy is circular with a circular skirt section, the area of the skirt can be obtained using the following expression:

$$
A_{S}=\frac{\pi}{4} \cdot\left(D_{S}^{2}-D_{B}^{2}\right)
$$

To obtain the radius of the buoy section, a representative skirt radius, $R_{S}$ is considered using Figure 5. This representatively provides an assumed definition to the position or locus where the application of the local drag loads is considered. Thus,

$$
R_{S}=\frac{\left(D_{S}+D_{B}\right)}{4}
$$


To obtain the width of the CALM buoy'skirt, the measurement is taken outer section of the skirt's rim to the outer region of the buoy's body, as expressed in Equation (5):

$$
W_{S}=\frac{\left(D_{S}-D_{B}\right)}{2}
$$

\subsubsection{Viscous Damping Load Computations}

A semi-empirical model including the drag term from Morison's formulation is used to calculate the viscous contributions in the CALM buoy heave and pitch damping [51]. From the description provided on the CALM buoy, the skirt's geometry and the load computation, it is possible to compute the load on a skirt segment. By bringing this force together using integration, the quadratic loads of heave and pitch damping are derived from the circumference of the skirt. The expression for the infinitesimally considered section of the skirt area where the local drag loads act, as provided in the literature [50,51], is:

$$
d A=\frac{\left(D_{S}^{2}-D_{B}^{2}\right)}{8} \cdot d \alpha
$$

It has been identified that the local velocity upon the skirt area, $d A$ is a function of the velocities for the pitch $(\theta)$, roll $(\varphi)$, and heave $(z)$ motion, and which is computed using:

$$
\begin{aligned}
V(t, \alpha) & =\dot{z}(t)+y(\alpha) \cdot \dot{\varphi}(t)-x(\alpha) \cdot \dot{\theta}(t) \\
d F(t, \alpha) & =-\frac{1}{2} \cdot \rho \cdot V(t, \alpha) \cdot|V(t, \alpha)| \cdot C_{D} \cdot d A
\end{aligned}
$$

The value for $C_{D}$ is the only empirical parameter in Equation (8) for the model for which a suitable value should be chosen. Keulegan and Carpenter [52] discovered that when the flow is oscillatory, the $C_{D}$, the dimensionless drag coefficient, may be affected by the amplitude of the motion. Thus, the dimensionless $K C$ number is frequently used to express the amplitude. In this study, it implies that the $C_{D}$ 's value may be influenced by the motion amplitude of the skirt part in question, as model-tested in MARIN [50,51]. In summary, the drag loads on the CALM buoy skirt that have been examined are flow related. At a sharp edge, it is believed that separation and formation of eddies will occur. The $C_{D}$ value is independent of the Re value. The independence of the Re was also mentioned by Sarpkaya \& O'Keefe [53] as a contribution in the instance of oscillating flow through sharp-edged plates.

\subsubsection{Damping Computations on Buoy}

The total frequency dependent damping is made up of a linear equivalent viscous term and a linear potential term when evaluating the CALM buoy damping in the frequency domain (FD). The quadratic drag loads on the skirt are represented by the viscous damping term $[50,51]$. As a corollary, it will be a dependent of the amplitude of the motion, as in Equation (9).

$$
B_{i i}(\omega)=B_{i i, P O T}(\omega)+B_{i i, V I S C}(\omega)
$$

For the heave damping, it is also assumed that the CALM buoy performs a harmonic motion, in the form given in Equation (10).

$$
z(t)=z_{a} \cdot \cos \omega t
$$

For the pitch damping, the $K C$ values considered in the literature [50,51], are given as:

$$
\begin{gathered}
K C=2 \pi \cdot \frac{z_{a}}{W_{s}} \\
K C=2 \pi \cdot \frac{R_{s} \cdot \theta_{a}}{W_{s}}
\end{gathered}
$$




\subsubsection{Force Computations on Buoy}

The forces that act on the buoy assume an irrotational motion and an ideal fluid and neglect the effect of viscosity; thus, they are calculated by the use of linear wave theory [54-57]. For small waves, the linearization of the dynamic free-surface boundary condition is assumed. For small buoys, an approximation can be carried out to determine the excitation force, while the diffracted wave is neglected [58]. However, for cylindrical shaped buoys, Froude-Krylov force can be calculated for its heave motion, as presented in Equation (13). In principle, Froude-Krylov force assumes an ideal flow, where the pressure field is undisturbed, by applying the linear airy wave theory.

$$
f_{F K}^{\text {heave }}(\omega)=2 \pi a \rho g \frac{J_{1}(k a)}{k}\left[\left(\frac{e^{-k d}}{1+e^{-2 k h}}\right)+\left(\frac{e^{k d}}{1+e^{2 k h}}\right)\right]
$$

where $a$ is the radius of the buoy, $h$ is the water depth, $d$ is the draft of the buoy, $k$ is the wave number, $2 \pi a$ is the circumference of the buoy, and $J_{1}$ is the first-order Bessel function.

The hydrostatic stiffness, $F_{h}$ for a cylindrical buoy is given by Equation (14); where $a$ is the radius of the buoy, $g$ is the gravitational constant, and $\varepsilon_{b}$ is a vector representing the translational degree of freedom of the buoy.

$$
F_{h}=\rho g \pi a^{2} \varepsilon_{b}
$$

The heaving and swaying amplitude motions of the buoy is a factor of its slenderness ratio, as columnar buoys have lesser area around the water line than cylindrical buoys. This was given by the study by Jiang et al. [59] and Newman [60] on the heave inherent period of the buoy, given by Equation (15):

$$
w_{o}=\sqrt{ }\left(\frac{\rho g A_{o}}{M}\right)
$$

where $w_{0}$ is the heave natural frequency of the buoy, $\rho$ is seawater density, $g$ is gravitational constant, $M$ is the mass of the buoy and $A_{o}$ is the waterline area of the buoy.

\subsection{FSI Formulation E Governing Equations}

The formulation for the fluid-structure interaction (FSI) and governing equations are presented in this section. The governing equations used in numerical modelling is based on applying Newton's 2nd law of motion, Morison's equation, hydrodynamic equations, Navier-Stokes equation and Continuity equation. Details on stability and motion equations exist in texts [61-65]. For irregular waves in the CFD model, the flow considered here is turbulent, thus we neglect the forces due to elasticity and the surface tension.

\subsubsection{Newton's 2nd Law of Motion}

The Newton's law of Equation is numerically presented in Equation (16), where the Newtonian Force, $F$ is the external load of the system, $C v$ is the viscous damping, $k$ is the spring constant, $k x$ is the elastic force component and $M a$ is the inertia of the system. The Newtonian Force is given by the sum of the inertia force of the system, the viscous damping load and the elastic force components (also called the stiffness load of the system).

$$
F=M a+C v+k x
$$

\subsubsection{Navier-Stokes Equations}

The rule of Navier-Stokes Equations included here are for thermo-fluid incidents directed by these governing equations, based on the laws of conservation. The Navier-Stokes (N-S) equations is the broadly applied mathematical model to examine changes in those properties during dynamic interactions, thermal interactions, and fluidic motions. The Navier-Stokes equations assume that the fluid, at the scale of interest, is a continuum, in other words is not made up of discrete particles but rather a continuous substance. Hence, 
the Navier-Stokes equations consists of three (3) conservation laws: a time-dependent continuity equation for conservation of mass, three time-dependent conservation of momentum equations and a time-dependent conservation of energy equation.

For fluid that is considered incompressible and non-Newtonian, the Navier-Stokes Equations are applied [66,67]. The summation of the body force, pressure gradient and viscous force make up the fluid inertia. This is given in Equations (17)-(19), where $P$ is the pressure, $\mu$ is the kinematic viscosity, $F_{x}$ is the body force per unit mass in $x$-direction, $F_{y}$ is the body force per unit mass in $y$-direction and $F_{z}$ is the body force per unit mass in $z$-direction.

$$
\begin{aligned}
\left(\frac{\partial u}{\partial t}+u \frac{\partial u}{\partial x}+v \frac{\partial u}{\partial y}+w \frac{\partial u}{\partial z}\right) & =F_{x}-\frac{1}{\rho} \frac{\partial P}{\partial x}+\mu\left(\frac{\partial^{2} u}{\partial x^{2}}+\frac{\partial^{2} u}{\partial y^{2}}+\frac{\partial^{2} u}{\partial z^{2}}\right) \\
\left(\frac{\partial v}{\partial t}+u \frac{\partial v}{\partial x}+v \frac{\partial v}{\partial y}+w \frac{\partial v}{\partial z}\right) & =F_{y}-\frac{1}{\rho} \frac{\partial P}{\partial y}+\mu\left(\frac{\partial^{2} v}{\partial x^{2}}+\frac{\partial^{2} v}{\partial y^{2}}+\frac{\partial^{2} v}{\partial z^{2}}\right) \\
\left(\frac{\partial w}{\partial t}+u \frac{\partial w}{\partial x}+v \frac{\partial w}{\partial y}+w \frac{\partial w}{\partial z}\right) & =F_{z}-\frac{1}{\rho} \frac{\partial P}{\partial z}+\mu\left(\frac{\partial^{2} w}{\partial x^{2}}+\frac{\partial^{2} w}{\partial y^{2}}+\frac{\partial^{2} w}{\partial z^{2}}\right)
\end{aligned}
$$

Vortexes are basically formed as a result of instabilities generated from flow separations, as they travel through the hull. The flow is assumed to be incompressible; i.e., the energy of the vortexes are allowed to continuously increase or damp away, depending on the situation.

\subsubsection{Continuity Equations}

The Euler equation for incompressible flow is presented in Equation (20). In this paper, the CFD study was carried out for incompressible unsteady flow using continuity equations [67]. The dimensionless vector form of the continuity equations can be written as:

$$
\frac{\partial\{u\}}{\partial t}+\{u\} . \nabla\{u\}+\{\nabla p\}-\frac{1}{R_{e}} \nabla^{2}\{u\}=0
$$

The equations used in the formulation of finite volume method for incompressible and unsteady flow which is based on Navier Stokes and continuity equations, expresses nonlinear dimensionless parameters in Cartesian coordinate, as expressed in Equations (21) and (22).

$$
\begin{gathered}
\frac{\partial \Phi}{\partial t}+\Phi \cdot \nabla \Phi=\frac{1}{R e} \nabla^{2} \Phi-\nabla p \\
\nabla \cdot \Phi=0
\end{gathered}
$$

where $\Phi$ is a non-dimensional velocity vector component, expressed in three directions; $u$, $v$ and $w$. The Reynolds number ' $R e^{\prime}$ is expressed in terms of the flow incidence velocity $U$, the fluid viscosity $v$, and the cylinder diameter $D$, as given in Equation (23):

$$
R e=U D / v
$$

where the Reynold's number $R e$ is a measure of the flow velocity, the column diameter, and the kinematic viscosity of water.

However, mathematically, the expression for drag force is:

$$
F_{d}=\frac{1}{2} \rho v^{2} C_{d} A
$$

Therefore, the hydrodynamic drag in the X-direction is calculated as:

$$
F_{d}=\frac{1}{2} \rho A U^{2} C_{d}
$$




$$
C_{d}=\frac{2 F_{d}^{i}}{\rho A U^{2}}
$$

However, considering the Keulegan Carpenter number, $K C$ [51,52] which is given in Equation (26), as a function of the frequency of the oscillating wave $f_{w}$, vortex shedding and vortex induced motion (VIM) can be measured, thus the surface wave becomes an important parameter.

$$
K C=U / f_{w} L
$$

\subsubsection{Morison's Equations}

Based on the forces on the risers, the Morison's equation was used, as it considers the wave forces acting on a cylinder, due to the relative motion of body immersed in the fluid $[68,69]$. Thus, it yields the sum of the Froude-Kyrov force $F_{F K}$, the hydrodynamic force of the fluid, $F_{H}$, and the drag force, $F_{D}$. Morison's equation is expressed in Equation (28):

$$
F=\rho V \dot{u}+\rho C_{a} V(\dot{u}-\dot{v})+\frac{1}{2} \rho C_{d} A(u-v)|u-v|
$$

where $V$ is the volume of the body, $A$ is the area of the body, $C_{d}$ is the drag coefficient, $C_{m}$ is the inertial force coefficient. The equation can be simplified, as the fluid force is equal to the sum of the drag force and the force of inertia, thus Equation (29):

$$
F=\Delta a_{w}+\rho C_{a} \Delta a_{r}+\frac{1}{2}+\rho C_{d} A V_{r}\left|V_{r}\right|
$$

The global design conducted in this investigation was carried out under irregular wave, and the damping was calculated using the modified Morison Equation [46].

$$
F=\rho V \dot{u}+\rho C_{a} D A\left(V_{r}\right)+\frac{1}{2} \rho C_{d} A\left(V_{r}\right)\left|V_{r}\right|
$$

where $V$ is the volume of the body, $A$ is the area of the body, $D$ is the diameter of the body, $C_{d}$ is the drag coefficient, $C_{a}$ is the added mass coefficient, $C_{m}$ is the inertial force coefficient, and the $V_{r}$ is the relative velocity of fluid particles.

\section{Numerical Model}

\subsection{Model Description}

The model is the numerical design of the CALM buoy, carried out in ANSYS Fluent. The effect of vortex flow around the buoy was also investigated using CFD in the present study. in the present study. Other comparatively-related researches on CALM buoys include experimental investigations that could be used to verify the flow behavior on buoys [26,27]. The present study was conducted using ANSYS Fluent R2 2020 [70-74], in 2D bounded walls, and in 3D. However, the results of the 2D study were only presented herein. The k-epsilon turbulence model was used to develop the 2D CFD model. In the model setup, the velocity specification method was based on magnitude normal to boundary. The reference frame was absolute and the Gauge Pressure of $0 \mathrm{~Pa}$ was considered. The maximum iterations used per time step were 2000, with a time step size of 0.01 for 250 time-steps. In the turbulence model, the turbulence intensity was set at $5 \%$ and turbulent viscosity ratio was 10, given in Table 1. The momentum input is taken in absolute reference frame. The turbulence model's momentum was considered in the inlet zone using the magnitude velocity specification method which is applied normal to the boundary.

\subsection{CFD Model}

The CFD model is a pressure-based transient CFD model that uses k-epsilon (2 equations) standard turbulence model, with standard wall functions applied in near-wall treatment. The solver applies absolute approximations in the velocity formulation in 2D plane, for $\mathrm{X}$ 
and $\mathrm{Y}$ axes. The corresponding planar velocities are $u$ and $v$, respectively. For the k-epsilon model, the model constants are given in Table 2.

Table 1. Velocity parameters for inlet and Under-relaxation factor for the CFD model.

\begin{tabular}{cc}
\hline Parameters & Under-Relaxation Factor \\
\hline Pressure & 0.3 \\
Density & 1 \\
Body Forces & 1 \\
Momentum & 0.7 \\
Turbulent Kinetic Energy & 0.8 \\
Turbulent Dissipation Rate & 0.8 \\
Turbulent Viscosity & 1 \\
Turbent Intensity & $5 \%$ \\
Turbulent Viscosity Ratio & 10 \\
\hline
\end{tabular}

Table 2. Parameters for the constants used in k-epsilon Model.

\begin{tabular}{cc}
\hline Parameters & Constants \\
\hline Cmu & 0.09 \\
CI-Epsilon & 1.44 \\
C2-Epsilon & 1.92 \\
TKE Prandtl Number & 1 \\
TDR Prandtl Number & 1.3 \\
\hline
\end{tabular}

\subsection{Mesh Details}

It was modelled in 2D as one body with surface area of $9721.5 \mathrm{~m}^{2}, 1$ face, 5 edges and 4 vertices. The domain surface body has 82,846 Domain Nodes and 81,313 Elements, with mesh as shown in Figure 6. The statistics for the mesh details of the CALM buoy model conducted in ANSYS Fluent can be seen in Table 3. It summarises the amount of meshed sections applied on the 2D CFD model.
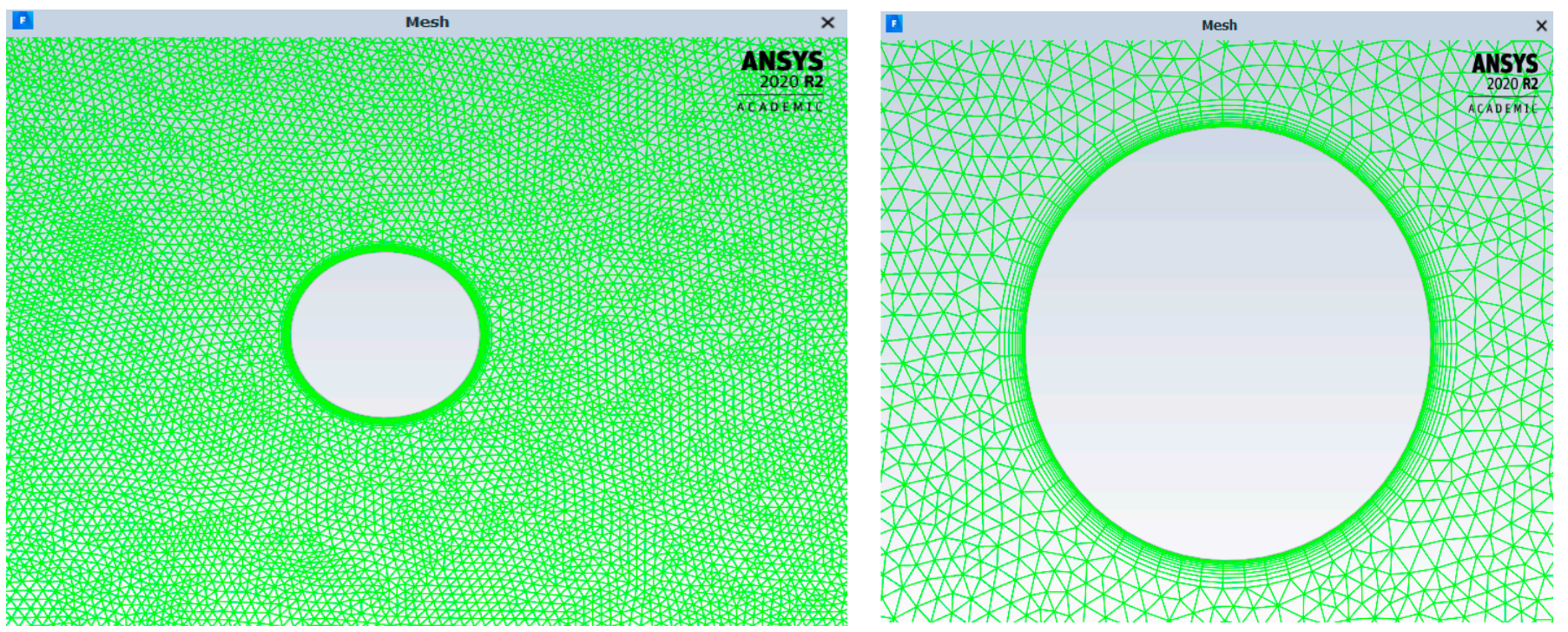

Figure 6. Mesh details showing two magnifications of the buoy model.

\subsection{Solution Method}

The solution method considered in the CFD modelling is pressure-velocity coupling. For the spatial discretization, the gradients were based on least square cell based gradient, second order pressure, second order upwind momentum, first order upwind turbulent kinetic energy, first order upwind turbulent dissipation rate and second order implicit 
turbulent dissipation rate. The result of the scaled residuals, with an absolute criterion of 0.01 is presented in Figure 7, for 34,000 iterations.

Table 3. Mesh statistics for the CFD model.

\begin{tabular}{cc}
\hline Parameters & Zone Type \\
\hline 81,313 mixed cells or elements & zone 2, binary \\
81,313 cell partition ids & zone 2, 2 partitions, binary \\
121,833 2D interior faces & zone 1, binary \\
560 2D wall faces & zone 5, binary \\
140 2D velocity-inlet faces & zone 6, binary \\
140 2D pressure-outlet faces & zone 7, binary \\
63 2D wall faces & zone 8, binary \\
41,423 nodes & binary \\
41,423 node flags & binary \\
\hline
\end{tabular}

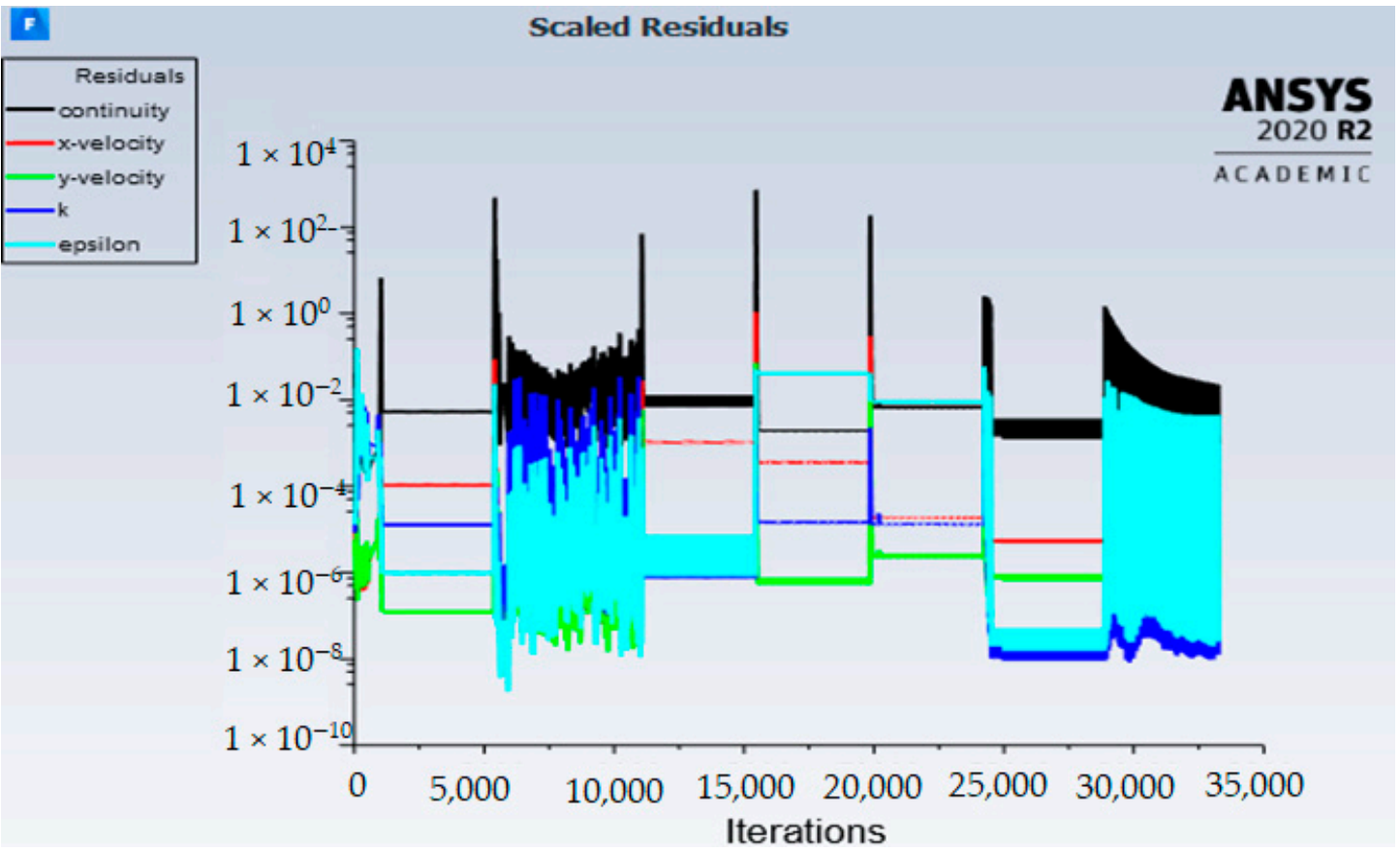

Figure 7. Convergence plot in ANSYS Fluent showing the scaled residuals.

\subsection{Boundary Conditions}

The boundary conditions considered for this CFD model are presented in Tables 4 and 5 . For the buoy boundary, it was set as stationary wall with a "no slip" shear condition. For the other 2 outer adjacent boundary walls, the specified shear condition was applied, using standard roughness model, roughness height of $0 \mathrm{~m}$ and roughness constant of $0.5 \mathrm{~m}$. The buoy setup in ANSYS CFD showing the boundary conditions is presented in Figure 8.

Table 4. Boundary conditions for the k-epsilon CFD 2D model.

\begin{tabular}{ccc}
\hline Parameters & Value & Unit \\
\hline Inlet Velocity & 1 & $\mathrm{~m} / \mathrm{s}$ \\
Outlet Pressure & 0 & $\mathrm{~Pa}$ \\
Wall & 0 & $\mathrm{~Pa}$ \\
\hline
\end{tabular}

\subsection{Materials \& Fluid Structure Interaction}

The CFD study shows fluid structure interaction (FSI) using ANSYS Fluent R2 2020. The buoy model was developed using aluminum and steel materials. The density for 
sea water is $1003 \mathrm{~kg} / \mathrm{m}^{3}$, the density for fresh water at normal temperature of $20{ }^{\circ} \mathrm{C}$ is $998.2 \mathrm{~kg} / \mathrm{m}^{3}$, the density of aluminum is $2719 \mathrm{~kg} / \mathrm{m}^{3}$, while the density of steel is $7800 \mathrm{~kg} / \mathrm{m}^{3}$. Details of the fluid properties considered are given in Table 6 . The parameters for the 2D CFD buoy model in ANSYS Fluent are given in Table 7 and Figure 9.

Table 5. Boundary physics showing boundaries and domain of the buoy in CFD.

\begin{tabular}{ccc}
\hline Domain & Boundaries & Boundary Type \\
\hline & Boundary: Buoy & Type: Wall \\
& Boundary: Inlet & Type: Velocity-Inlet \\
& Boundary: Outlet & Type: Pressure-Outlet \\
Surface Body & Type: Symmetry \\
& Boundary: Symmetry 1 & Type: Symmetry \\
& Boundary: Wall & Type: Wall \\
\hline
\end{tabular}

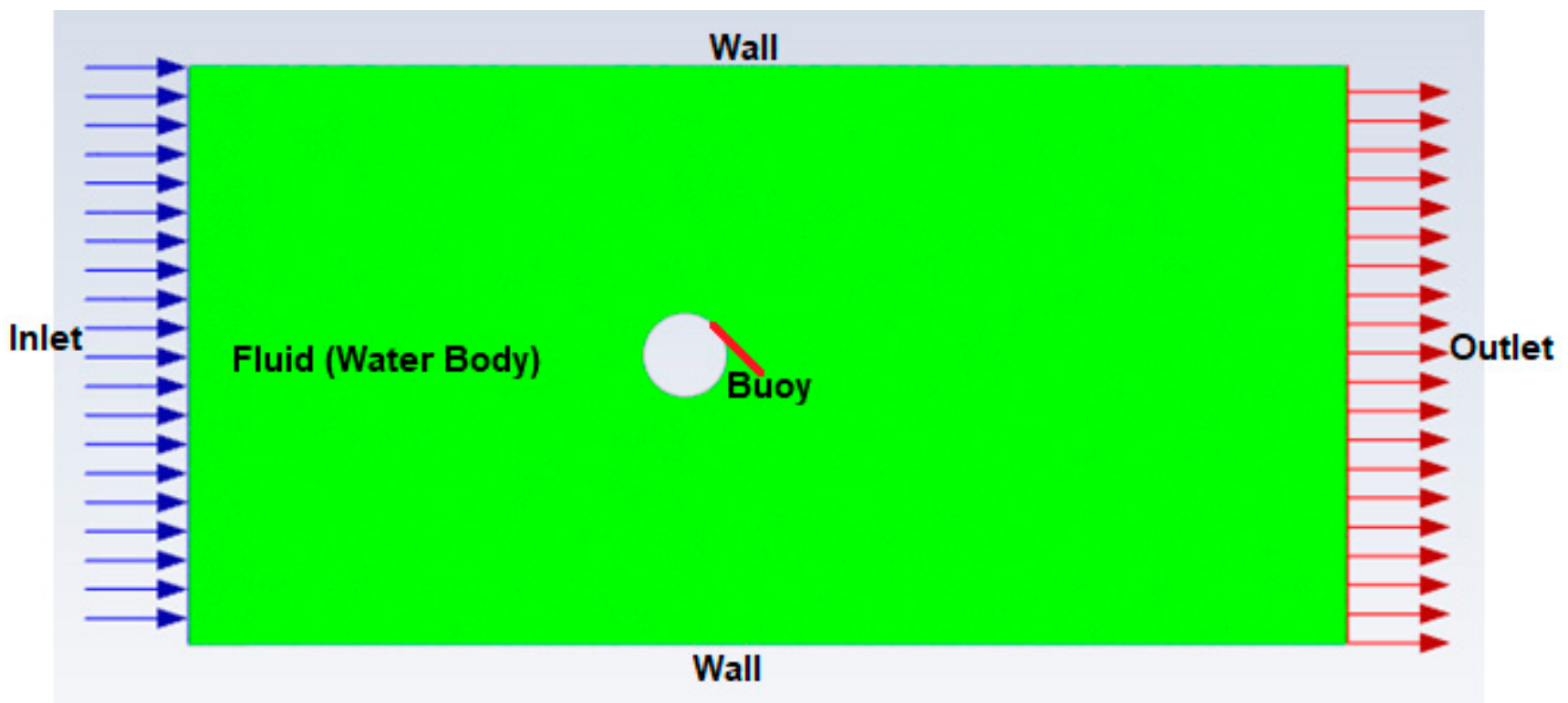

Figure 8. The setup of the buoy in ANSYS CFD showing boundary conditions.

Table 6. Properties of fluid (sea water) used in the FSI model in ANSYS Fluent.

\begin{tabular}{ccc}
\hline Parameters & Value & Unit \\
\hline Area & 1 & $\mathrm{~m}^{2}$ \\
Density of Air & 1.225 & $\mathrm{Kg} / \mathrm{m}^{3}$ \\
Density of Water & 998.2 & $\mathrm{Kg} / \mathrm{m}^{3}$ \\
Depth & 1 & $\mathrm{~m}$ \\
Length & 1 & $\mathrm{~m}$ \\
Atm. air Pressure & 0 & $\mathrm{~Pa}$ \\
Temperature & 288.16 & $\mathrm{~K}$ \\
Velocity of Air & 70 & $\mathrm{~m} / \mathrm{s}$ \\
Viscosity of Air & $1.7894 \times 10^{-5}$ & $\mathrm{Kg} / \mathrm{m} . \mathrm{s}$ \\
Viscosity of Water & 0.001003 & $\mathrm{Kg} / \mathrm{m} . \mathrm{s}$ \\
\hline
\end{tabular}

Table 7. Parameters for 2D CFD buoy model in ANSYS Fluent.

\begin{tabular}{ccc}
\hline Parameters & Value & Unit \\
\hline Buoy Diameter (D1) & 10 & $\mathrm{~m}$ \\
Horizontal Height of boundary near inlet to centre of buoy (H4) & 60 & $\mathrm{~m}$ \\
Horizontal Height of boundary near outlet (H5) & 80 & $\mathrm{~m}$ \\
Vertical height of boundary from top wall to centre of buoy (V2) & 35 & $\mathrm{~m}$ \\
Vertical height of boundary from top wall to centre of buoy (V3) & 35 & $\mathrm{~m}$ \\
\hline
\end{tabular}




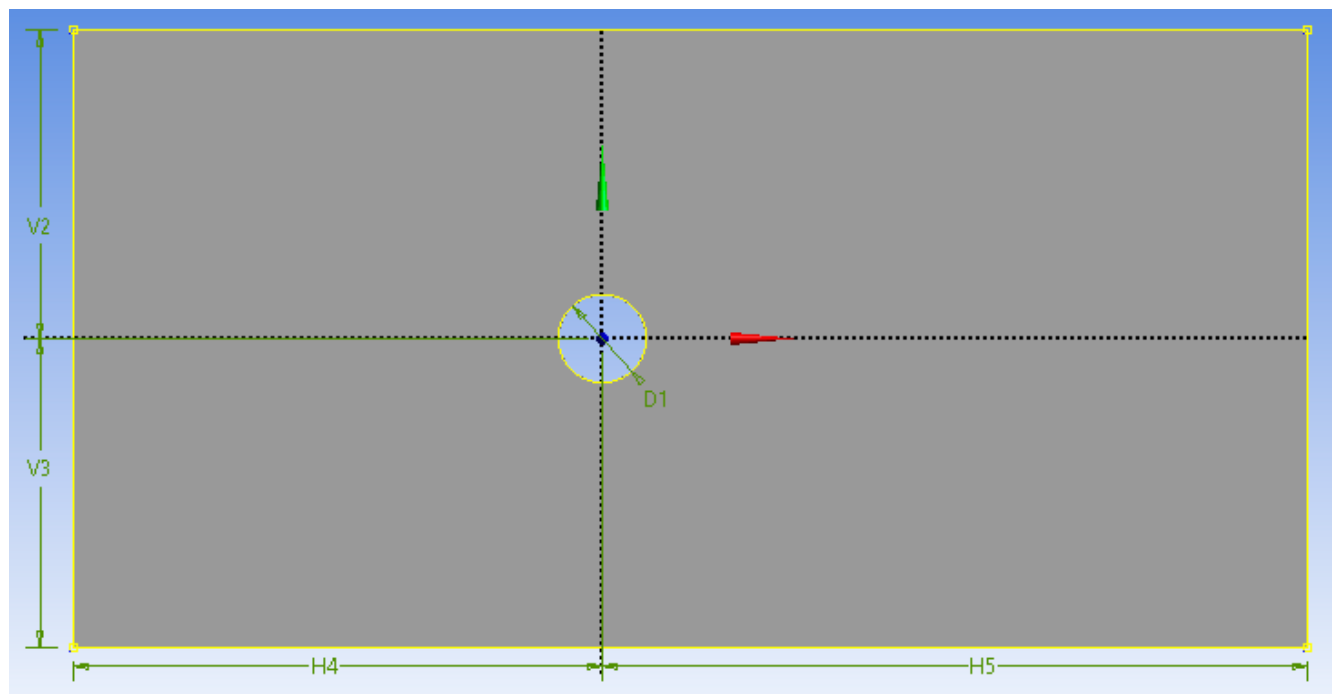

Figure 9. The parameters of the 2D CALM buoy model setup in ANSYS Fluent.

\section{Result and Discussion}

The results and discussion of the CFD study are presented in this section.

\subsection{Results of Flow Vorticity around Buoy}

From the result obtained from the CFD study, it can be observed that the pressure and velocity have an effect on the profile of the flow around the CALM buoy. The resulting profile in Figures 10 and 11 shows the velocity profiles for the flow around the CALM buoy. It can be observed that the flow from the inlet (LHS) moves towards the outlet (RHS) of the wall. The flow creates different flow patterns, depending on the force filed developed around the structure when the velocity is $0.45 \mathrm{~m} / \mathrm{s}, 1.0 \mathrm{~m} / \mathrm{s}$ and $10 \mathrm{~m} / \mathrm{s}$. This speed is chosen based on the environmental condition used in investigating the flow characteristics and motion behaviour. Another CFD model was carried out in ANSYS Fluent to investigate the sloshing effect of water waves on the CALM buoy. A total of 2 different velocities, of magnitudes $0.45 \mathrm{~m} / \mathrm{s}$ and $1.5 \mathrm{~m} / \mathrm{s}$, were investigated for the CFD analysis of the CALM buoy, as they represent 2 different ocean conditions. This is confirmed in the streamline series for the velocity along directions in Figures 12 and 13. In the velocity contours in Figures 10 and 11, it can be observed that the higher the velocity, the higher the vorticity around the CALM buoy.

\subsection{Results of Vortex Effect from the Flow Regimes}

The investigation of the vortex effect on the buoy was conducted using different flow regimes as shown in Figure 11. It was studied using 3 different cases of velocities: $0.45 \mathrm{~m} / \mathrm{s}$ for normal operation condition, $1.0 \mathrm{~m} / \mathrm{s}$ for extreme weather condition and $10 \mathrm{~m} / \mathrm{s}$ for survival weather condition. Due to the waves generated on the buoy, there was some ripple effect from viscous damping on the buoy. It was also noticed that there was a higher vortex flow on the buoy under a higher velocity profile, which is attributed to contributions from linear and quadratic damping from the buoy motion responses in the heave, roll and pitch motions. This can be seen in the streamline series in Figures 12 and 13.

Based on the generated linear contributions partially resulting from radiated waves and the frictional viscous effect, it can be opined that the buoy has some eddies in the direction of the flow. On the other hand, the generated quadratic contributions partially resulting from the eddies separating the buoy's vertices, and the sharp edges around the buoy's skirt, it was found to develop much higher buildup of ripple-like vortices. Hence, these buildups result in some vortex effect on the buoy, however, further studies are recommended on postprocessing the vortex-induced motion (VIM) of the buoy. 


\subsection{Results of Pressure, Velocity and Wall Shear Profiles on the Buoy}

The investigation of the pressure, velocity and wall shear profiles on the buoy were conducted using different flow regimes in Figures 14-19. In Figures 14 and 15, the pressure profile can be seen to be higher with higher magnitude of velocity as seen for $0.45 \mathrm{~m} / \mathrm{s}$ case is higher than the $1.5 \mathrm{~m} / \mathrm{s}$ case. Similarly in Figures 16 and 17, the velocity profile can be seen to be higher with higher magnitude of velocity as seen for $0.45 \mathrm{~m} / \mathrm{s}$ case is higher than the $1.50 \mathrm{~m} / \mathrm{s}$ case. In Figures 18 and 19, the wall shear can be seen to have highest distribution in a ripple for $0.45 \mathrm{~m} / \mathrm{s}$ case which is higher than the $1.50 \mathrm{~m} / \mathrm{s}$ case.

In this investigation, the highest velocity distribution for $0.45 \mathrm{~m} / \mathrm{s}$ case is $1.963 \mathrm{~m} / \mathrm{s}$ in Figure 14, while the highest velocity distribution for $1.50 \mathrm{~m} / \mathrm{s}$ case is $2.611 \mathrm{~m} / \mathrm{s}$ in Figure 15 . Moreover, the highest-pressure distribution for $0.45 \mathrm{~m} / \mathrm{s}$ case is $12.18 \mathrm{~Pa}$ in Figure 16, while the highest-pressure distribution for $1.50 \mathrm{~m} / \mathrm{s}$ case is $5.766 \mathrm{~Pa}$ in Figure 17. Lastly, the highest wall shear distribution for $0.45 \mathrm{~m} / \mathrm{s}$ case is $0.5809 \mathrm{~Pa}$ in Figure 18, while the highest wall shear distribution for $1.50 \mathrm{~m} / \mathrm{s}$ case is $0.5963 \mathrm{~Pa}$ in Figure 19. In this investigation, the wall shear, pressure, and velocity profiles reflect some vorticity patterns in the axial direction which differed under different cases of velocity magnitudes.
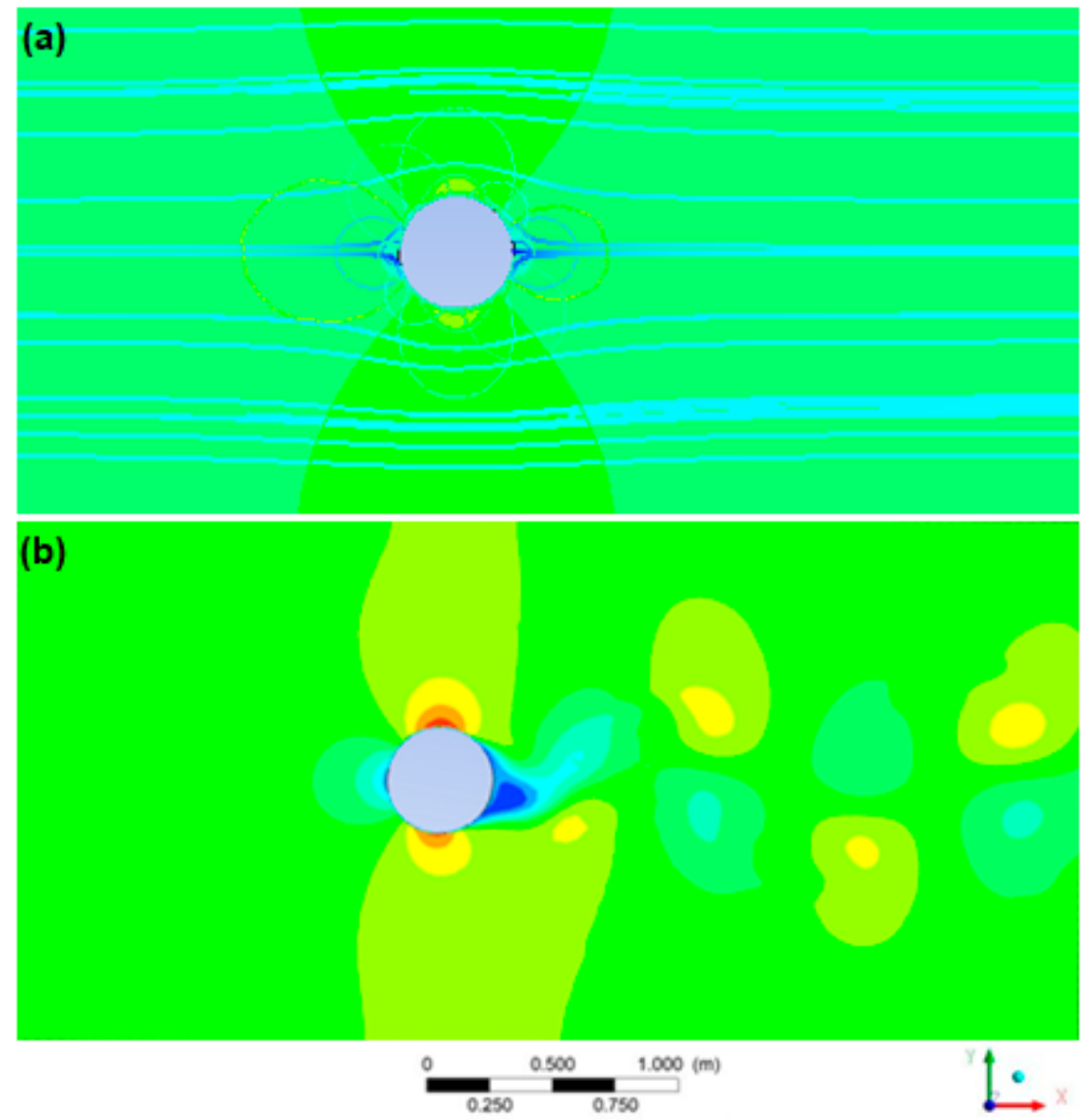

Figure 10. Velocity u Contour profile for Vortex effect around the CALM Buoy for (a) $0.45 \mathrm{~m} / \mathrm{s}$ flow velocity with streamlines and (b) $1.5 \mathrm{~m} / \mathrm{s}$ flow velocity without streamlines, in ANSYS Fluent.

\subsection{Results of Turbulence Streamlines}

The numerical calculation for the buoy profiles for different parameters to present individual turbulence streamlines are conducted in this sub-section. In Figure 20, the streamline series for the velocity cases is higher in $1.0 \mathrm{~m} / \mathrm{s}$ compared to the $0.45 \mathrm{~m} / \mathrm{s}$ across the $x$-axis and $y$-axis. Furthermore, in Figure 21, the streamline series for the pressure cases is higher in $1.0 \mathrm{~m} / \mathrm{s}$ compared to the $0.45 \mathrm{~m} / \mathrm{s}$ across the $x$-axis and $y$-axis. Similarly in Figure 22, the streamline series for the turbulence kinetic energy cases is higher in $1.0 \mathrm{~m} / \mathrm{s}$ compared to the $0.45 \mathrm{~m} / \mathrm{s}$ across the $x$-axis and $y$-axis. These results on turbulence kinetic 
energy show that the turbulence model has an influence on the buoy model in this CFD study. This implies that the parameters of the turbulence model can be used to improve the performance of the buoy model.

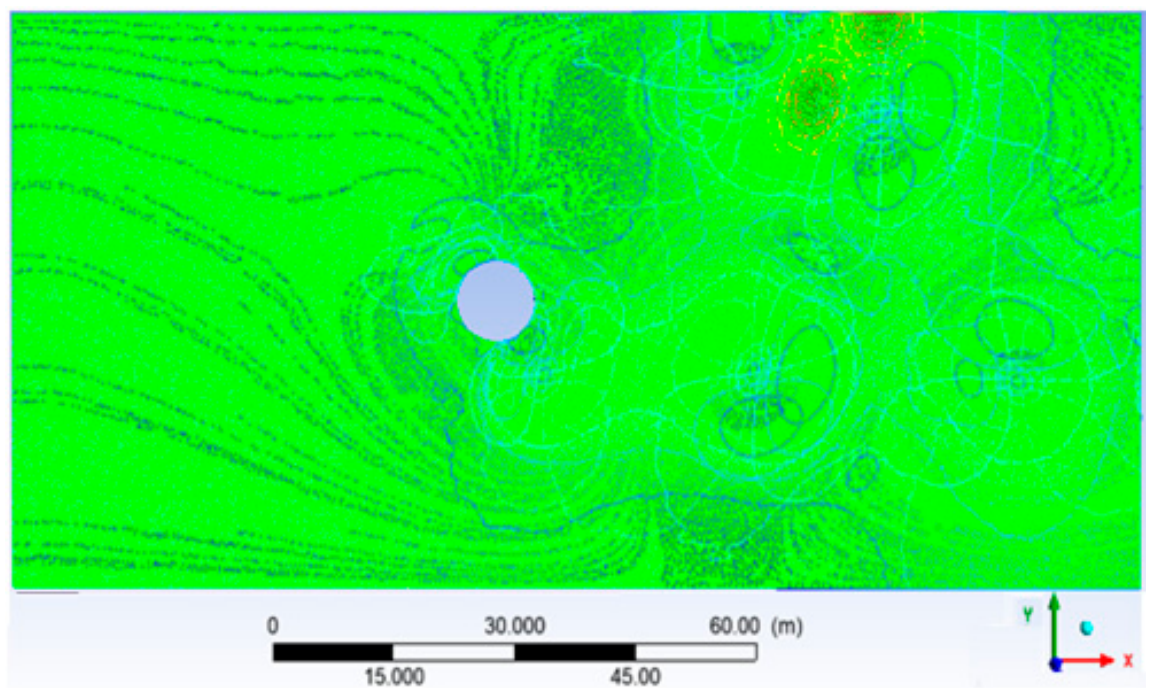

Figure 11. Vortex effect on the free-floating buoy showing flowlines and fluid-structure interaction.

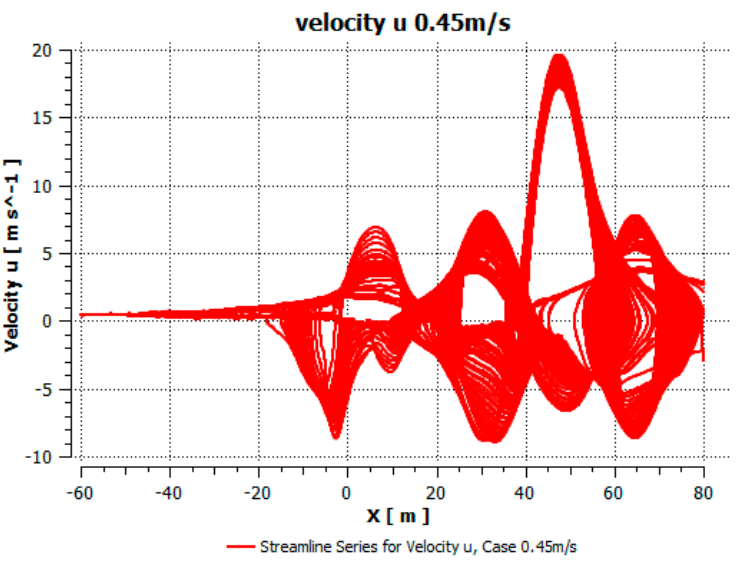

(a)

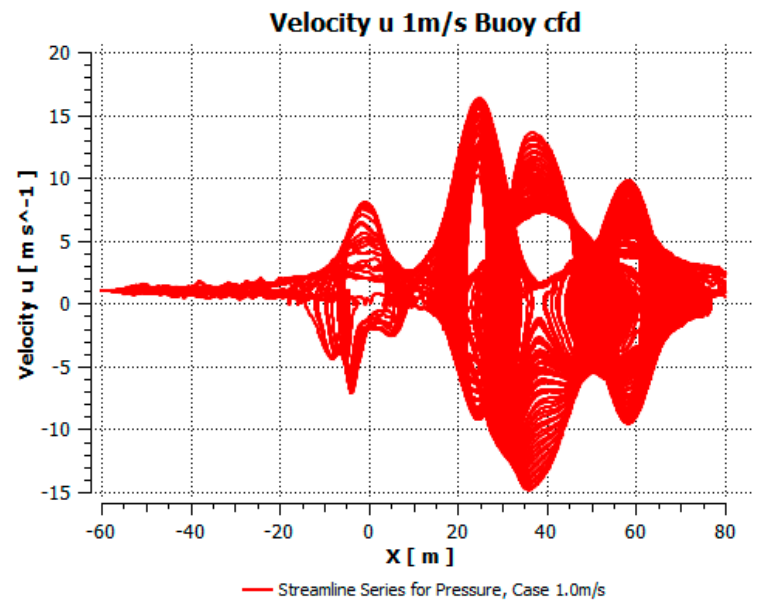

(c)

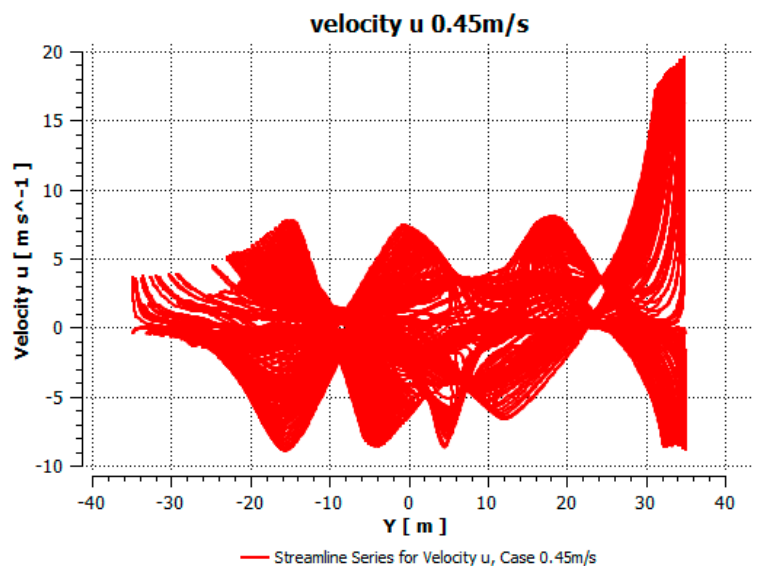

(b)

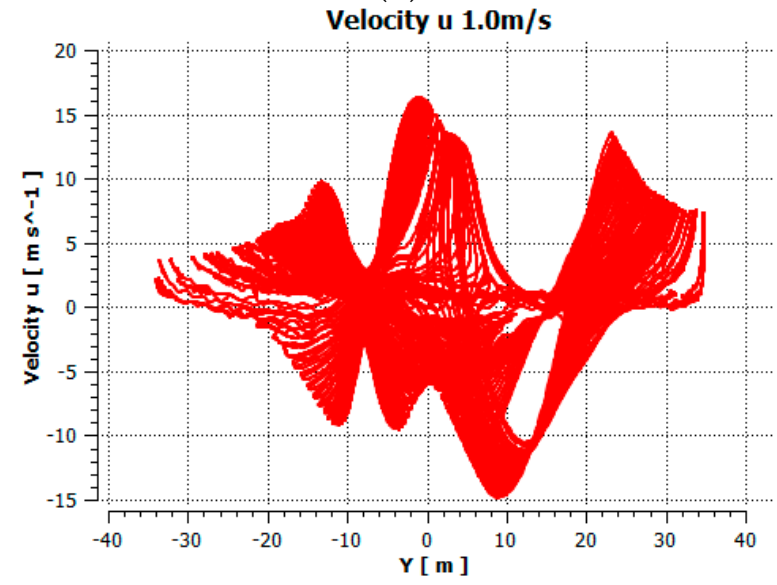

— Streamline Series for Velocity u, Case $1.0 \mathrm{~m} / \mathrm{s}$

(d)

Figure 12. Velocity profile in Axial direction $(\mathrm{u})$ for the flow on the buoy showing the streamline series: $(\mathbf{a}, \mathbf{b})$ Streamline series for Velocity $\mathrm{u}$, Case $0.45 \mathrm{~m} / \mathrm{s}$; (c,d) Streamline series for Velocity $\mathrm{u}$, Case $1.0 \mathrm{~m} / \mathrm{s}$. 


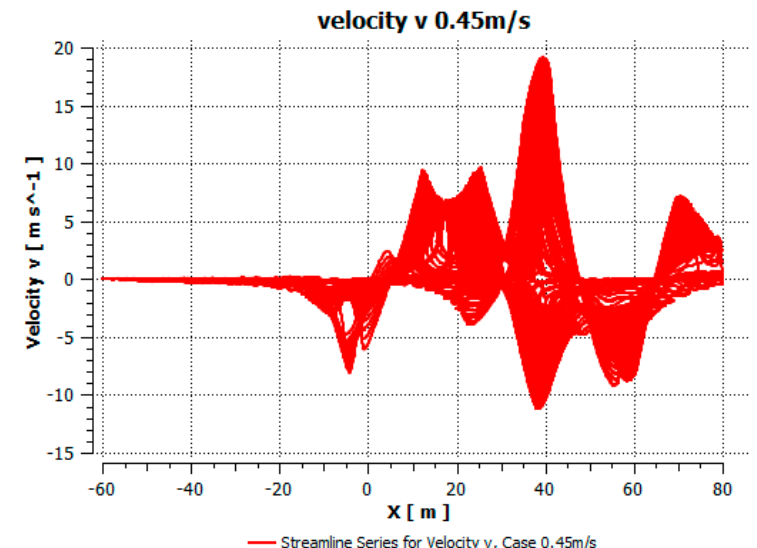

(a)

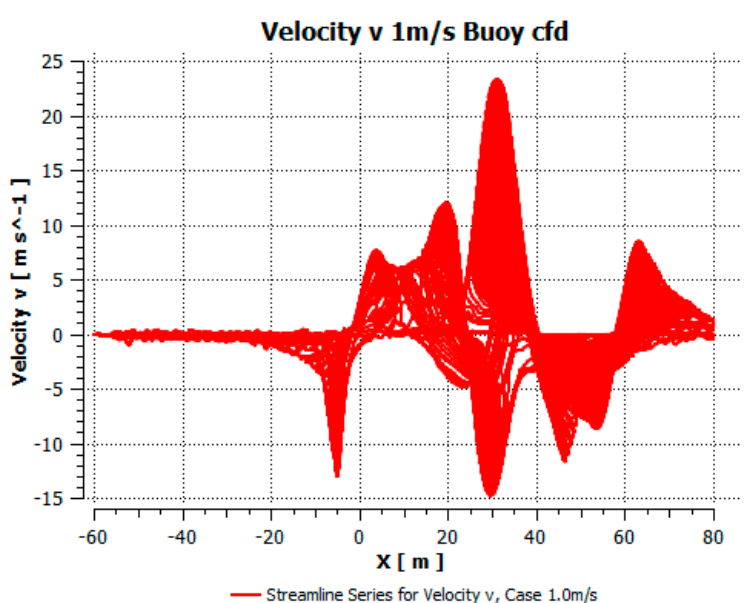

(c)

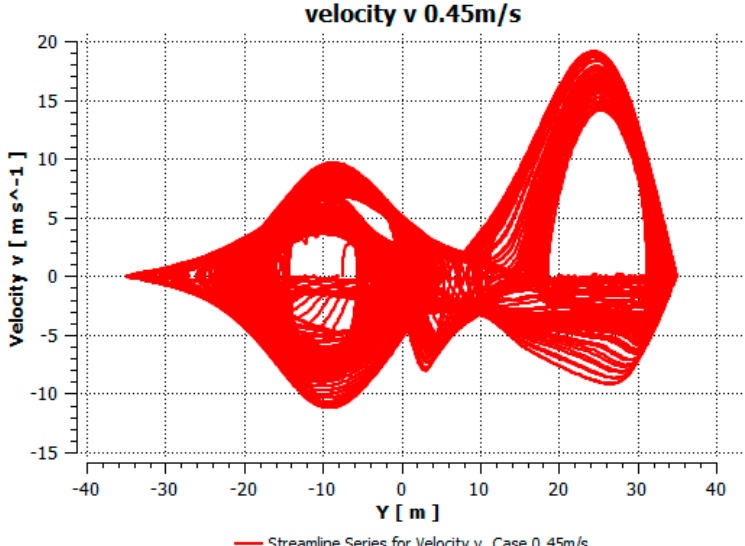

(b)

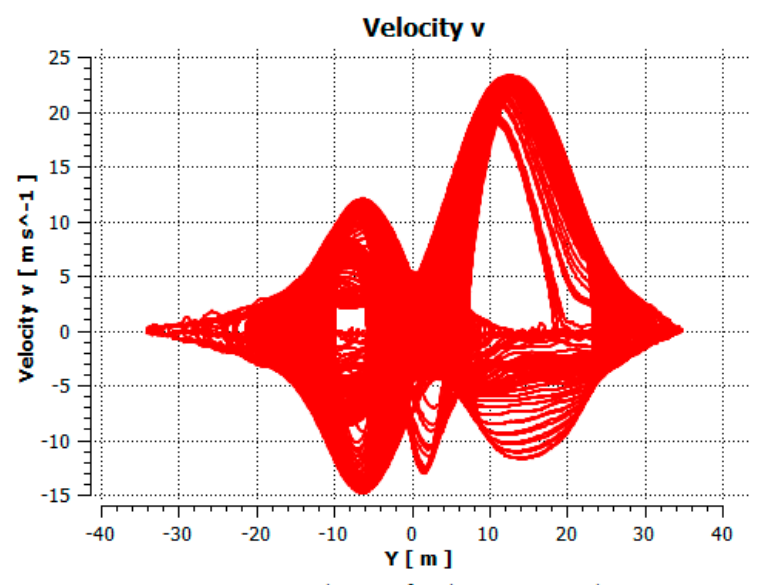

(d)

Figure 13. Velocity profile in Vertical direction $(\mathrm{v})$ for the flow on the buoy showing the streamline series: $(\mathbf{a}, \mathbf{b})$ Streamline series for Velocity v, Case $0.45 \mathrm{~m} / \mathrm{s}$; (c,d) Streamline series for Velocity v, Case $1.0 \mathrm{~m} / \mathrm{s}$.

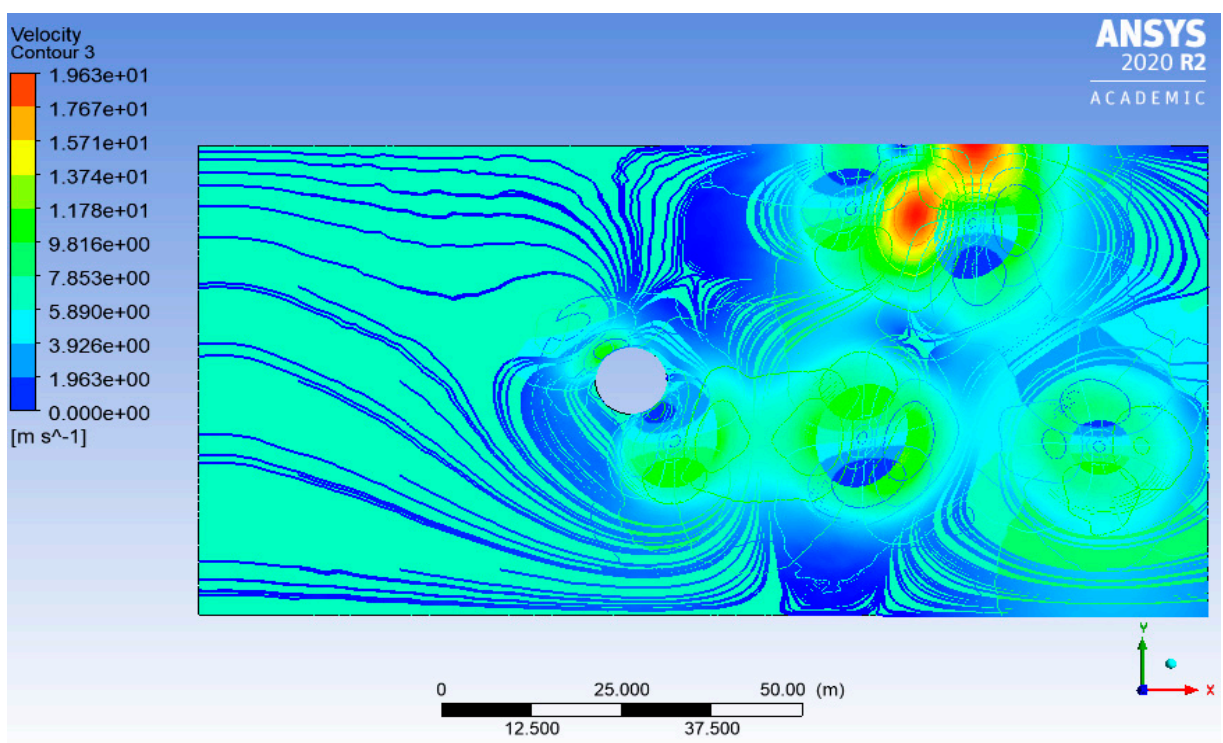

Figure 14. Velocity contour for the flow on the free-floating buoy under $0.45 \mathrm{~m} / \mathrm{s}$ case. 


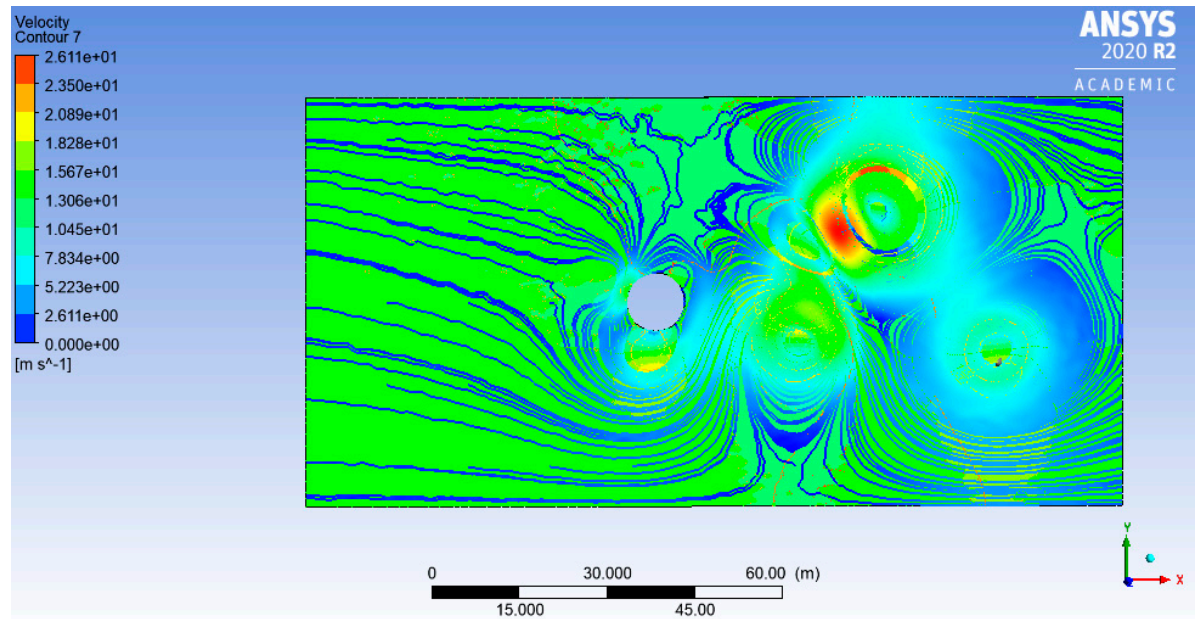

Figure 15. Velocity contour for the flow on the free-floating buoy under $1.5 \mathrm{~m} / \mathrm{s}$ case.

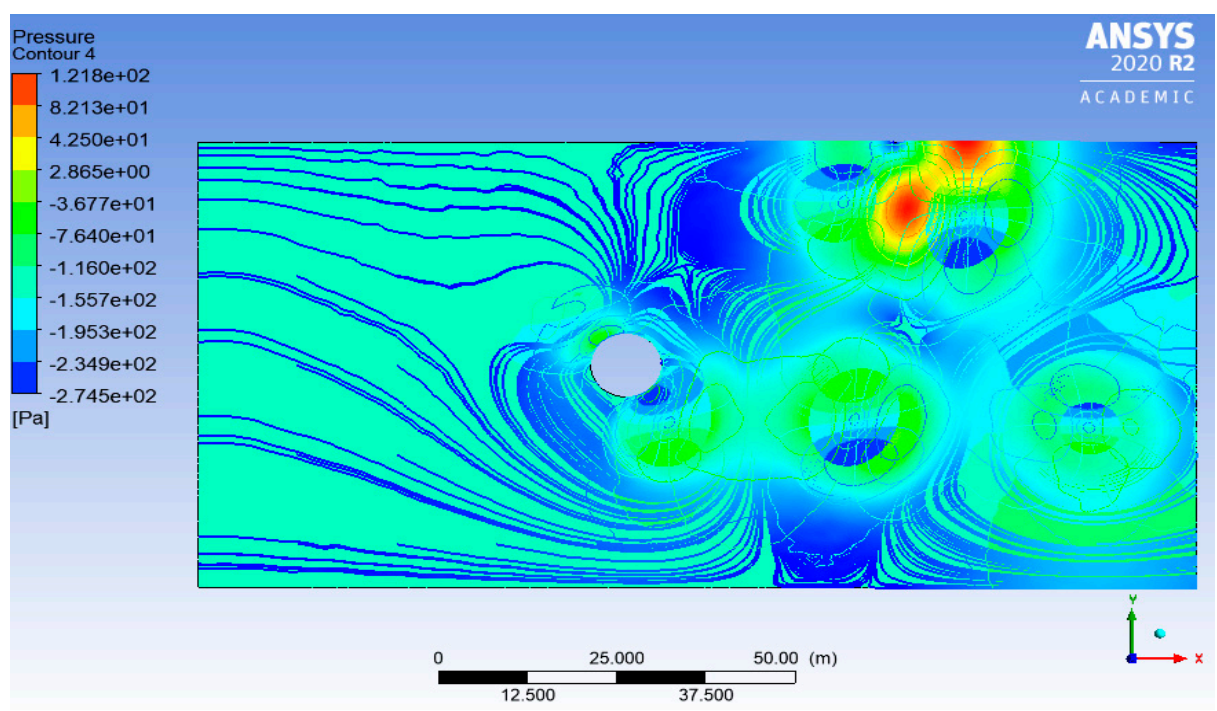

Figure 16. Pressure contour for the flow on the free-floating buoy under $0.45 \mathrm{~m} / \mathrm{s}$ case.

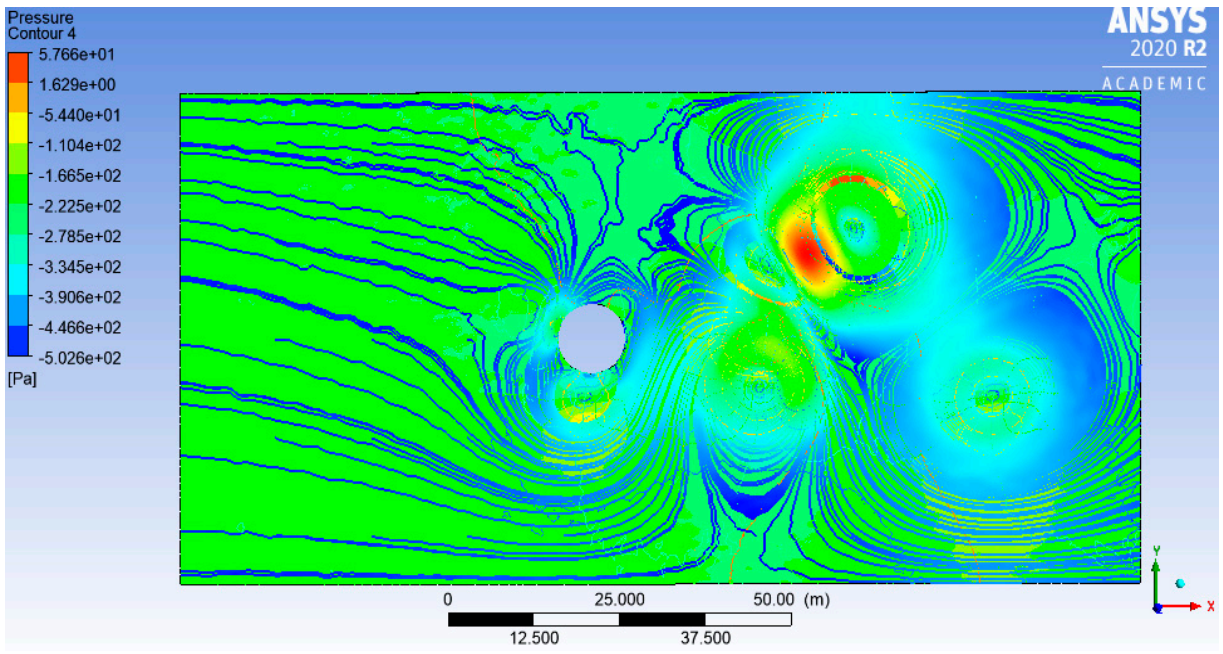

Figure 17. Pressure contour for the flow on the free-floating buoy under $1.5 \mathrm{~m} / \mathrm{s}$ case. 


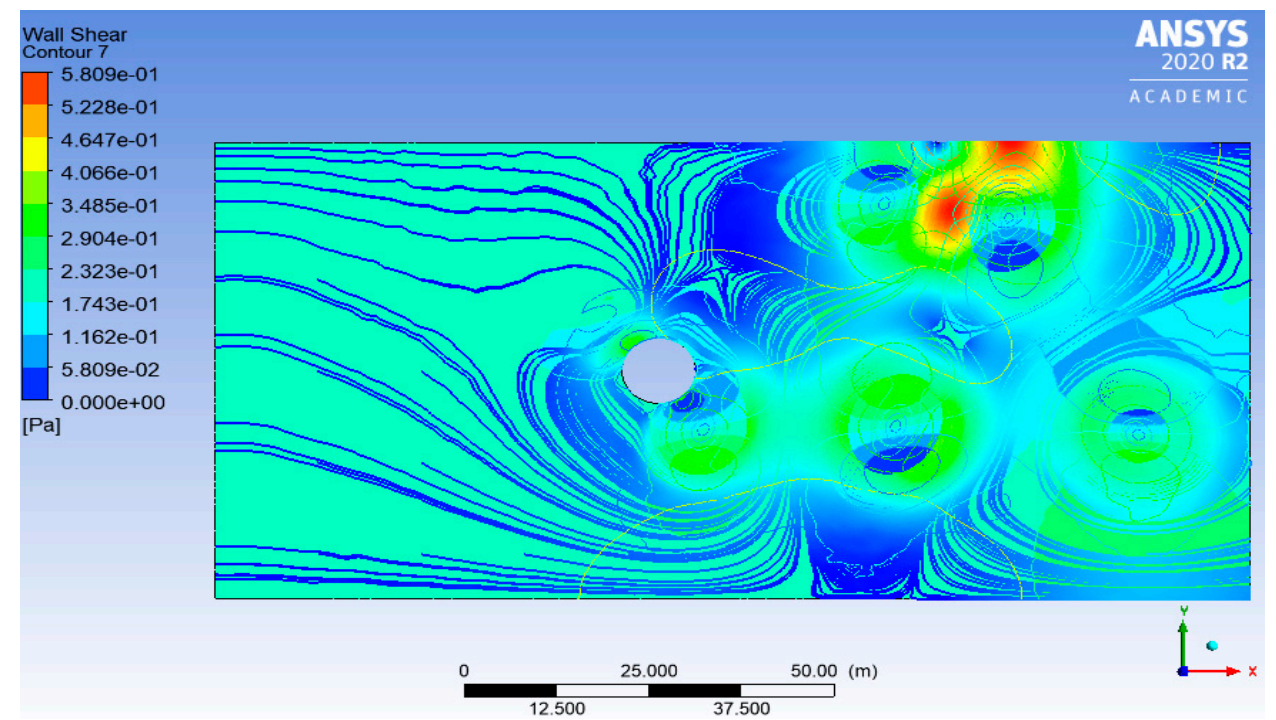

Figure 18. Wall shear contour for the flow on the free-floating buoy under case $0.45 \mathrm{~m} / \mathrm{s}$.

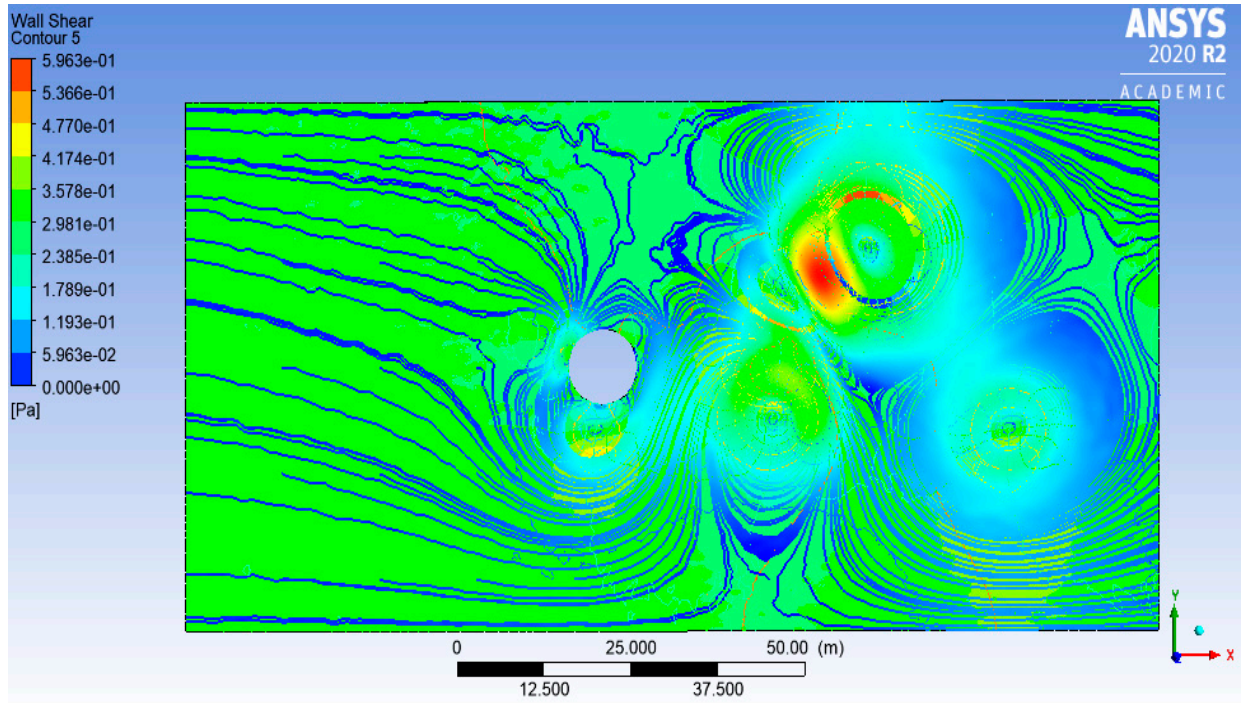

Figure 19. Wall shear contour for the flow on the free-floating buoy under $1.5 \mathrm{~m} / \mathrm{s}$ case.

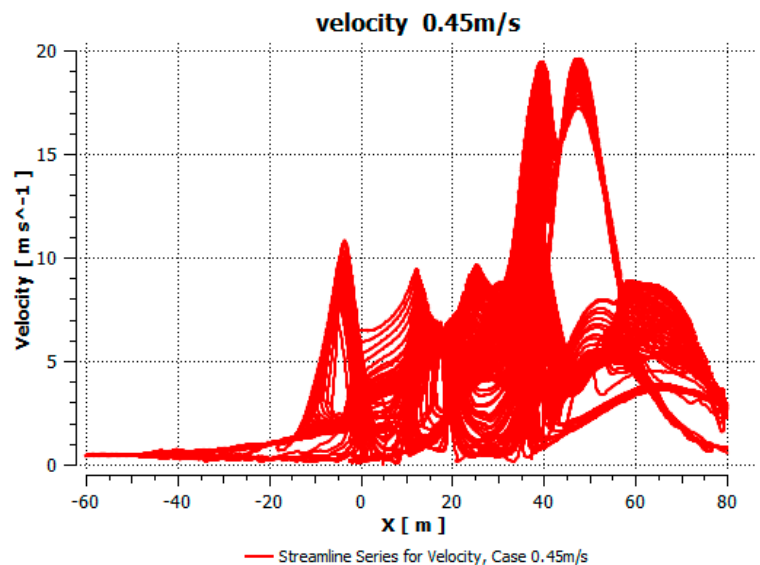

(a)

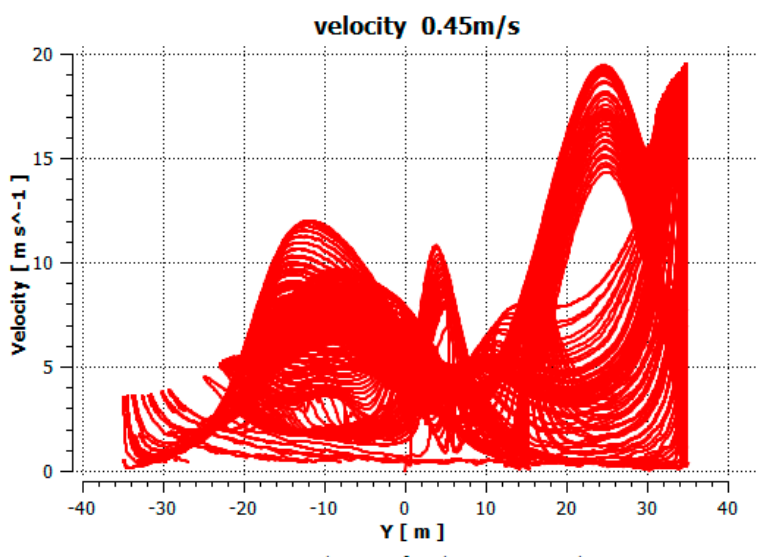

— Streamline Series for Velocity, Case $0.45 \mathrm{~m} / \mathrm{s}$

(b)

Figure 20. Cont. 


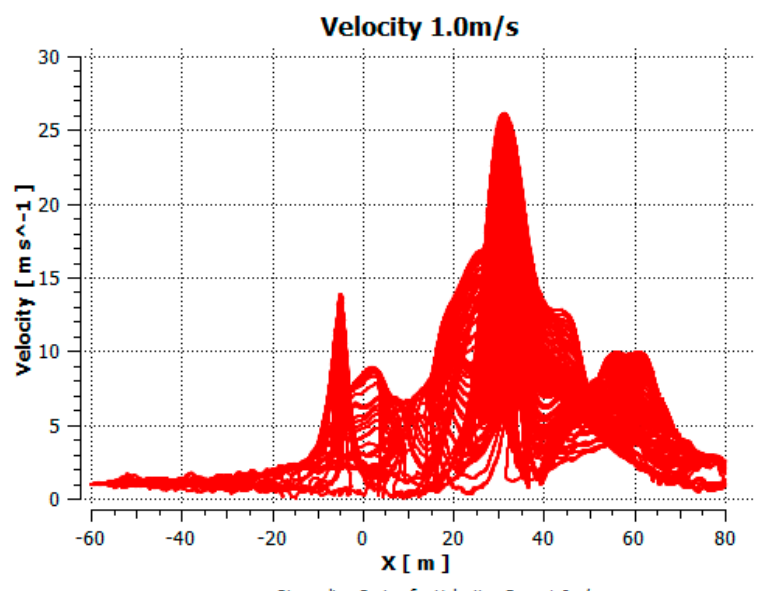

(c)

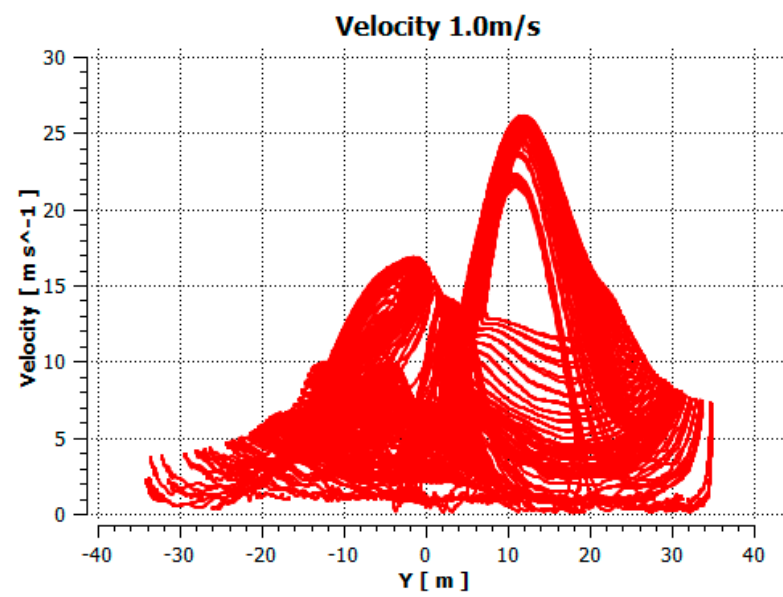

— Streamline Series for Velocity, Case $1.0 \mathrm{~m} / \mathrm{s}$

(d)

Figure 20. Velocity profile for the flow on the buoy showing the streamline series: $(\mathbf{a}, \mathbf{b})$ Streamline series for Velocity, case $0.45 \mathrm{~m} / \mathrm{s}$; (c,d) Streamline series for Velocity, case $1.0 \mathrm{~m} / \mathrm{s}$.

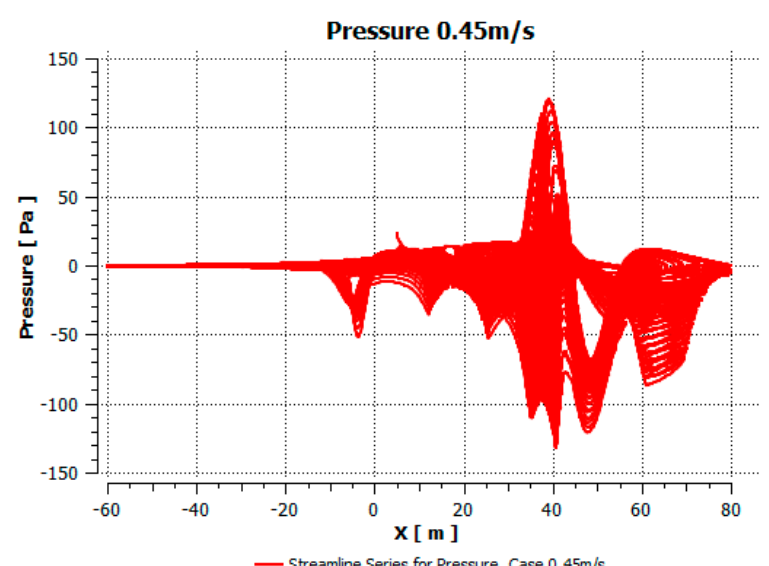

(a)

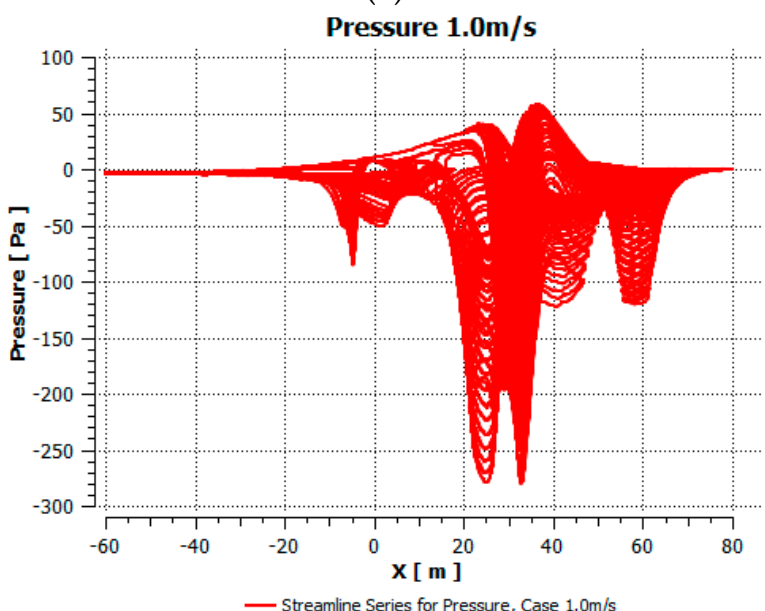

(c)

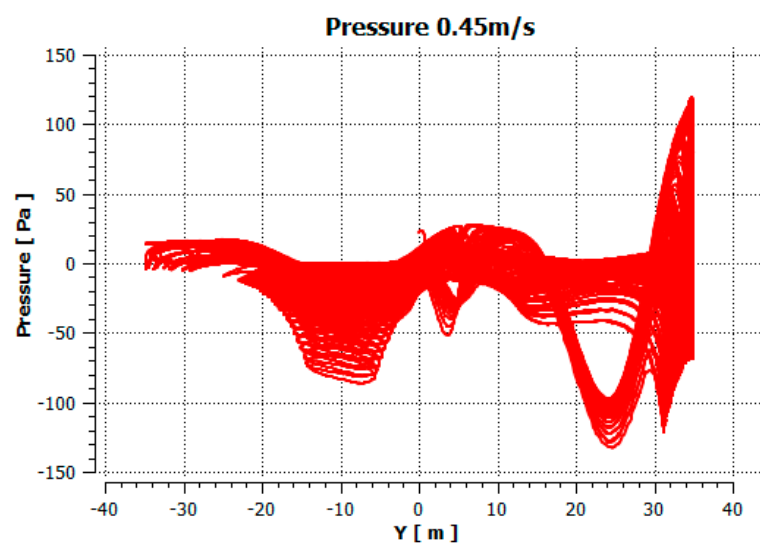

— Streamline Series for Pressure, Case $0.45 \mathrm{~m} / \mathrm{s}$

(b)

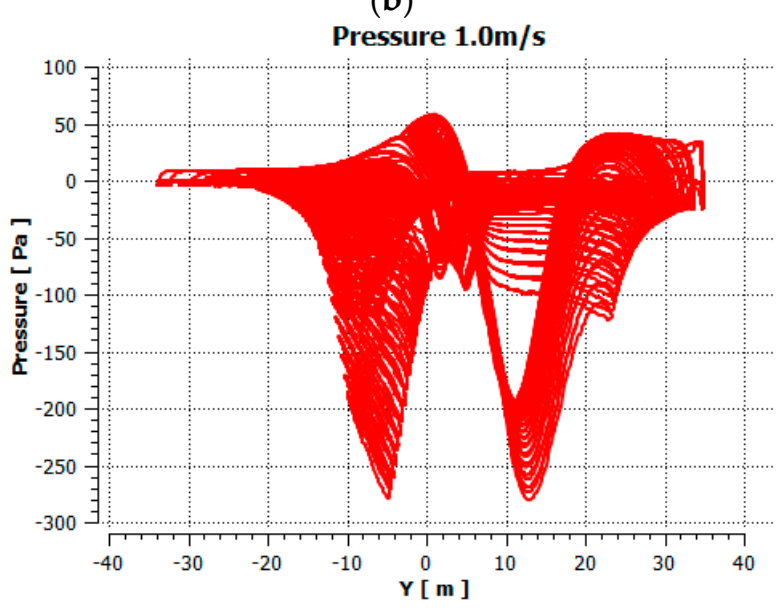

— Streamline Series for Pressure, Case $1.0 \mathrm{~m} / \mathrm{s}$

(d)

Figure 21. Pressure profile for the flow on the buoy showing the streamline series: $(\mathbf{a}, \mathbf{b})$ Streamline series for Pressure, case $0.45 \mathrm{~m} / \mathrm{s}$; (c,d) Streamline series for Pressure, case $1.0 \mathrm{~m} / \mathrm{s}$. 


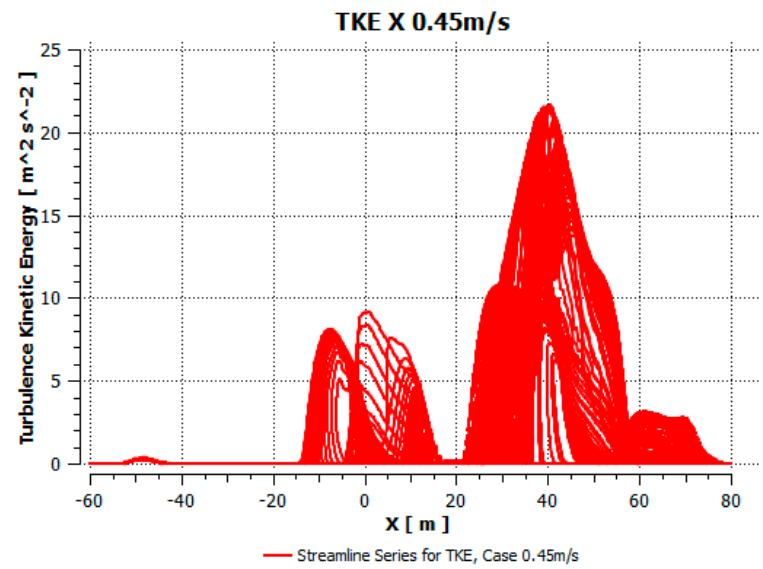

(a)

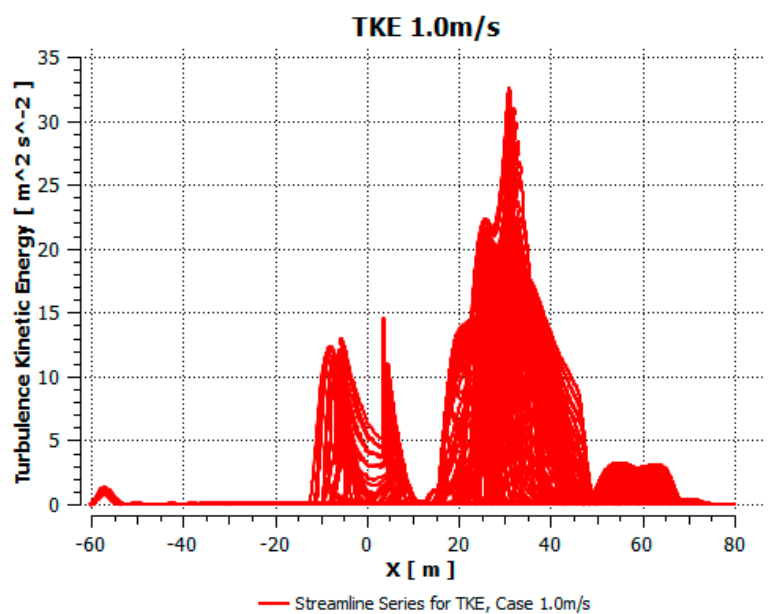

(c)

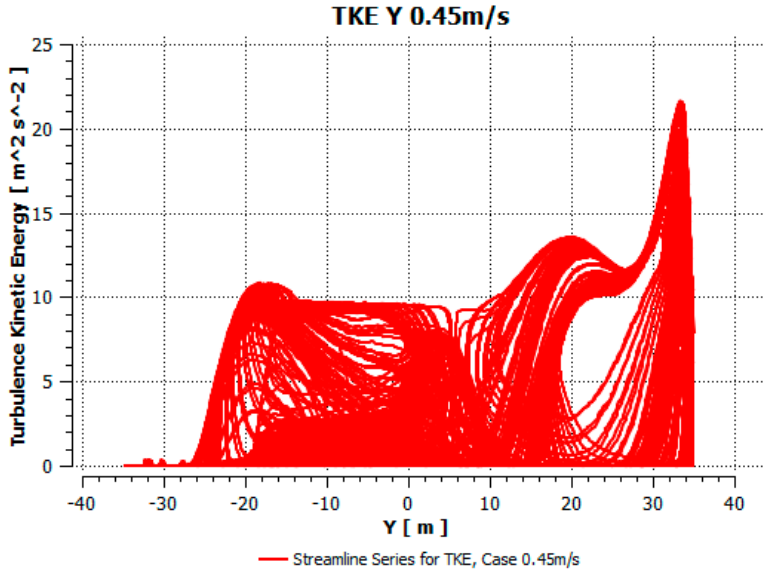

(b)

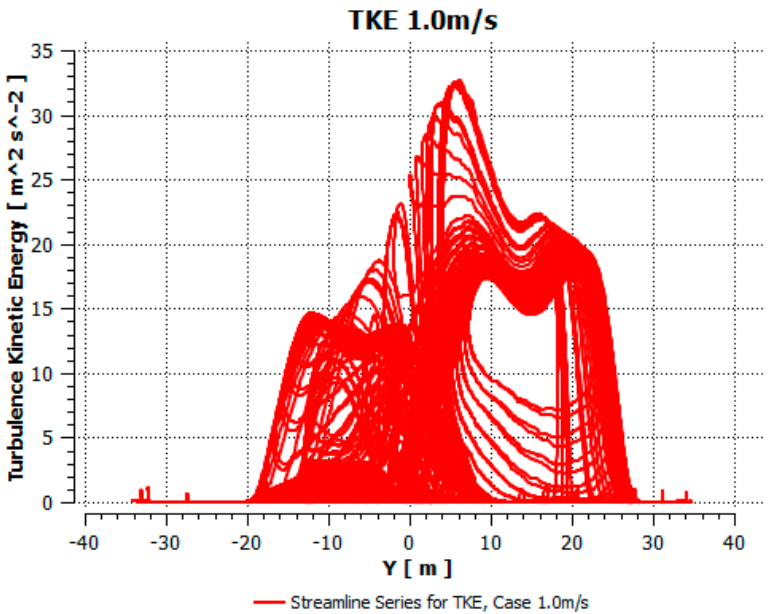

(d)

Figure 22. Turbulence profile for the flow on the buoy showing the streamline series: $(\mathbf{a}, \mathbf{b})$ Streamline series for Turbulence, case $0.45 \mathrm{~m} / \mathrm{s}$; (c,d) Streamline series for Turbulence, case $1.0 \mathrm{~m} / \mathrm{s}$.

\subsection{Results of Viscous Damping}

The calculation for the viscous damping is a very important aspect of the modelling. In this present investigation, damping estimation was considered for the CALM buoy. In principle, there are quadratic and linear contributions on damping from the buoy motion responses in the heave, roll and pitch motions. The generation of the linear contributions partly result from radiated waves and the frictional viscous effect. Conversely, the generation of the quadratic contributions are partly resulting from the eddies that separate the buoy's vertices, and the sharp edges around the buoy's skirt. Using a semi-empirical model, using MARIN's viscous study on CALM buoy [50,51], the values for the viscous damping coefficients are obtained. The viscous damping is proportional to a single drag coefficient, skirt plate geometry, wave frequency, velocity, and buoy amplitude. To compute this viscous damping semi-empirically, some model variations and parameters are considered, as given in Table 8. The semi-empirical method for viscous damping is detailed in literature [6,51]. The results are compared with the prediction in the present study, as seen in Figure 23. However, detailed computations are not given in this paper.

In the study by Cozijn et al. [51], a comparison was performed between the damping values recorded in the forced oscillation experiments and the damping values computed using the pitch and heave damping model for the CALM buoy having a skirt. The drag coefficient $C_{D}$ employed in the heave and pitch damping model was chosen to match the measured damping values as closely as possible. This strategy, however, can only be employed when model test data is available. In some circumstances, a different approach 
to determining a suitable value for the drag coefficient $C_{D}$ is required. Empirically, the $C_{D}$ values are examined in greater depth by using Equations (27) and (28) to compute for the $C_{D}$ value for the CALM buoy's skirt from each pitch and heave oscillation test result. The accompanying $K C$ numbers, which are defined in the equations, can then be displayed as a function of the $C_{D}$ values.

Table 8. Model parameters used in Empirical study for Viscous Damping.

\begin{tabular}{cccccc}
\hline Model & $\begin{array}{c}\text { Metacentric } \\
\text { Height }(\mathbf{m})\end{array}$ & $\begin{array}{c}\text { Buoy Diameter } \\
(\mathbf{m})\end{array}$ & $\begin{array}{c}\text { Buoy Skirt } \\
\text { Diameter }(\mathbf{m})\end{array}$ & $\begin{array}{c}\text { Buoy Skirt } \\
\text { Width }(\mathbf{m})\end{array}$ & Responses ** \\
\hline A1 & 0.25 & 10.00 & 13.90 & 0.1 & $\mathrm{LF}+\mathrm{WF}$ \\
A2 & 0.25 & 10.00 & 13.90 & 0.2 & $\mathrm{LF}+\mathrm{WF}$ \\
A3 & 0.25 & 10.00 & 13.90 & 0.3 & $\mathrm{LF}+\mathrm{WF}$ \\
B1 & 0.25 & 10.00 & 13.90 & 0.4 & $\mathrm{LF}+\mathrm{WF}$ \\
B2 & 0.25 & 10.00 & 13.90 & 0.5 & $\mathrm{LF}+\mathrm{WF}$ \\
B3 & 0.25 & 10.00 & 14.90 & 1.0 & $\mathrm{LF}+\mathrm{WF}$ \\
C1 & 0.25 & 10.00 & 15.90 & 1.5 & $\mathrm{LF}+\mathrm{WF}$ \\
C2 & 0.25 & 10.00 & 16.90 & 2.0 & $\mathrm{LF}+\mathrm{WF}$ \\
C3 & 0.25 & 10.00 & 17.90 & 2.5 & $\mathrm{LF}+\mathrm{WF}$ \\
D1 & 0.25 & 10.00 & 13.90 & 1.5 & $\mathrm{LF}+\mathrm{WF}$ \\
D2 & 0.50 & 10.00 & 13.90 & 1.5 & $\mathrm{LF}+\mathrm{WF}$ \\
D3 & 0.75 & 10.00 & 13.90 & 1.5 & $\mathrm{LF}+\mathrm{WF}$ \\
\hline
\end{tabular}

Note $^{* *}$ : LF-Low Frequency heading, WF-Wave Frequency.

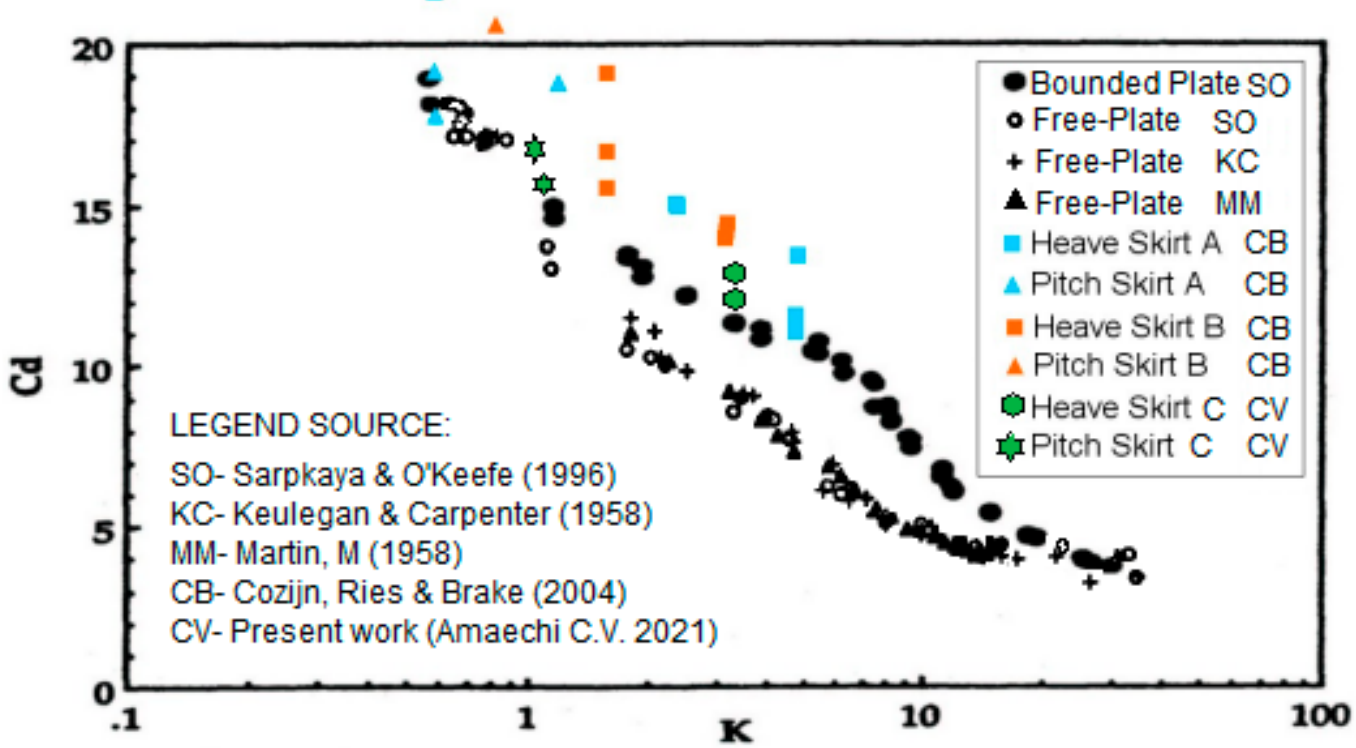

Figure 23. Drag coefficient versus Keulegan-Carpenter number for wall-bounded 2D plates and free rectangular 2D plates. (Please note that all the points are approximate interpolations. Also, the original graph was made by Sarpkaya and O'Keefe [53], as they created the black and white image, based on their own experiments with wall mounted plates in a flume tank. Cozijn et al. [51] added the orange and blue points, which are the data resulting from MARIN's forced oscillation model experiments with a CALM buoy. Amaechi C.V. [26] added the green points from experiments using WITmotion bluetooth sensors on CALM buoy in Lancaster University wave tank. The image was adapted with permissions of ISOPE, MARIN and ASME. Original sources: $[26,51,53])$.

Some studies were conducted on perforated plates, free plates, and bounded plates $[52,53,75-77]$. Sarpkaya \& $\mathrm{O}^{\prime}$ Keefe [53] gave $C_{D}$ values for a wall bounded plate in a $2 \mathrm{D}$ oscillating flow as a function of the $K C$ number. Figure 23 depicts similar findings in a graph (black dots) from 2 publications $[51,53]$. The $C_{D}$ values obtained from the heave and pitch forced oscillation tests for the CALM buoy skirt are presented in the same figure (coloured points) for comparison. The CALM buoy skirt $C_{D}$ values are identical to the 
$C_{D}$ values for a wall-bounded plate in a $2 \mathrm{D}$ oscillating flow, as shown in Figure 23. The CALM buoy skirt $C_{D}$ values, on the other hand, appear to be slightly greater than the ones reported by Sarpkaya \& O'Keefe [53]. This can be explained by the fact that, despite the same flow patterns, the flow around the buoy skirt is axi-symmetric 3D rather than $2 \mathrm{D}$. It is also feasible that the drag loads on the CALM buoy bilge are not insignificant. In such instance, their contribution to total drag is included in the drag loads on the skirt in the study presented here, which could lead to an overestimation of the $C_{D}$ values. Recent applications have been conducted by coupling CALM buoy models using related hydrodynamic formulations in literature [78-81].

\section{Conclusions}

In this research, the CALM buoy was numerically investigated under water waves using computational fluid dynamics (CFD) to investigate the vortex effect on the buoy. Some mathematical modelling and governing equations for the CALM buoy system were presented. By considering the complexity of a CALM buoy system, the boundary conditions were formed using some assumptions and some governing equations. Considerations were made using the damping to develop the equations by considering the buoy and its skirt. However, special attention is given to the CALM buoy and the skirt in the formulation. For the CFD study, the model was conducted using a 2D model. The results showed peculiar characteristics, which should be considered in the design due to the drag and damping implications on it. This research also presents findings from the vortex effect, velocity, pressure, wall shear and turbulence. This study is important to enable designers to design appropriately based on the CALM buoy system, buoy geometry and CFD data.

The model highlights include the following: firstly, a theoretical model is presented on motion characterization for CALM buoy model without attached hoses. Secondly, the CALM buoy model was conducted on the vortex effect in 2D CFD under different parameters. Thirdly, different novel techniques were applied in the numerical investigation to obtain the influence of the turbulence model on the CALM buoy. Fourthly, the study on the motion scenario from pressure, velocity, wall shear, and motion response study on waves upon the CALM buoy. Lastly, prediction of the turbulence and flow vorticity on the CALM buoy's motion characteristics was presented from the study.

The study presented buoy motion profiles based on 2D CFD study and numerical and theoretical predictions. From an offshore mechanical point of view, the motion characterisation phenomenon has been confirmed to exist as a result of the response from the water waves and other global loads on the CALM buoy. The study shows more dimension on the CALM buoy in a water body and buoy motion. The study has presented a comprehensive formulation of the offshore structure as is necessary for understanding the stability and dynamics behaviour. The vortex flow effect on the free-floating buoy is investigated using CFD. Another validation is recommended on an engineering application by coupling using the Orcaflex FEM to prove it is a working application of the mathematical formulations presented herein. However, further studies are recommended on the CFD study on buoy motion with moorings based on the analytical approximations for the moving boundary of marine hoses, and investigation on hose-snaking behaviour.

Author Contributions: Conceptualization, C.V.A. and J.Y.; methodology, C.V.A. and J.Y.; software, C.V.A. and J.Y.; validation, C.V.A. and J.Y.; formal analysis, C.V.A. and J.Y.; investigation, C.V.A. and J.Y.; resources, C.V.A.; data curation, C.V.A.; writing-original draft preparation, C.V.A.; writingreview and editing, C.V.A. and J.Y.; visualization, C.V.A.; supervision, C.V.A. and J.Y.; project administration, C.V.A. and J.Y.; funding acquisition, C.V.A. and J.Y. All authors have read and agreed to the published version of the manuscript.

Funding: The Department of Engineering, Lancaster University, UK and Engineering and Physical Sciences Research Council (EPSRC)'s Doctoral Training Centre (DTC), UK are highly appreciated. In addition, the funding of Overseas Postgraduate Scholarship by Niger Delta Development Commission (NDDC), Port Harcourt, Nigeria is also appreciated, as well as the support of Standards Organisation 
of Nigeria (SON), F.C.T. Abuja, Nigeria. The article processing charges (APC) for this article was funded by the 1st Author-C.V.A., with support from MDPI's Inventions.

Institutional Review Board Statement: Not applicable.

Informed Consent Statement: Not applicable.

Data Availability Statement: The data supporting the reported results cannot be shared at this time, as it is been used in producing more publications on this research.

Acknowledgments: The author acknowledges the technical support from Lancaster University Engineering Department, especially during the COVID-19 pandemic. The permission obtained from Wayne Herbrich of SOFEC Inc. to use the image in Figure 2 is appreciated. The permission for the original source for Figure 23 was also requested from ASME publishers. The permissions obtained from Cozijn Hans of MARIN and Jin S. Chung of ISOPE to use the image in Figure 23 are also appreciated. The authors also acknowledge the feedback given on this submission by George Aggidis of Lancaster University UK and Long-Yuan Li of Plymouth University, UK. The authors appreciate the support of the reviewers and journal editors in reviewing this manuscript.

Conflicts of Interest: The authors declare no conflict of interest. The funders had no role in the design of the study; in the collection, analyses, or interpretation of data; in the writing of the manuscript, or in the decision to publish the results.

\section{Abbreviations}

2D Two Dimensional

3D Three Dimensional

6DoF Six Degrees of Freedom

As Area of the skirt

ABS American Bureau of Shipping

API American Petroleum Institute

ASME American Society of Mechanical Engineers

CALM Catenary Anchor Leg Mooring

CB Cylindrical Buoy

$\mathrm{C}_{\mathrm{D}} \quad$ Dimensionless Drag Coefficient

CFD Computational Fluid Dynamics

CMS Conventional Mooring Systems

$\mathrm{C}_{\mathrm{V}} \quad$ Viscous damping

$\mathrm{D}_{\mathrm{B}} \quad$ Diameter of the buoy

$D_{S} \quad$ Diameter of the skirt

DNVGL Det Norkse Veritas \& Germanischer Lloyd

EU European Union

FANS Finite Element Model

$\mathrm{F}_{\mathrm{D}} \quad$ drag force

FD frequency domain

FEM Finite-Analytic Navier-Stokes

$\mathrm{F}_{\mathrm{FK}} \quad$ Froude-Kyrov force

$\mathrm{F}_{\mathrm{H}} \quad$ Hydrodynamic force of the fluid

FOS Floating Offshore Structure

FPSO Floating Production Storage and Offloading

FSI Fluid Structure Interaction

FSO Floating Storage and Offloading

ID Inner Diameter

IEFG Interpolating Element Free Galerkin

ISOPE International Society of Offshore and Polar Engineering

JIP Joint Industry Project

LF Low Frequency

LHS Left Hand Side

MSL Mean Sea Level

OD Outer Diameter

PLEM Pipeline End Manifold 


$\begin{array}{ll}\text { RAO } & \text { Response Amplitude Operator } \\ \text { RHS } & \text { Right Hand Side } \\ \text { R }_{\mathrm{S}} & \text { Representative radius of skirt } \\ \text { SALM } & \text { Single Anchor Leg Moorings } \\ \text { SON } & \text { Standards Organisation of Nigeria } \\ \text { SPM } & \text { Single Point Mooring } \\ \text { VIM } & \text { Vortex-Induced Motion } \\ \text { VoF } & \text { Volume of Fluid } \\ \text { WF } & \text { Wave Frequency } \\ \text { WIM } & \text { Wave-Induced Motion }\end{array}$

\section{References}

1. He, N.; Zhang, C.; Kang, Z. Analysis of Coupling Characteristics of the Offloading Buoy System in West Africa Seas. In Proceedings of the 28th International Ocean and Polar Engineering Conference, Sapporo, Japan, 10-15 June 2018. Available online: https:/ / onepetro.org/ISOPEIOPEC/proceedings-abstract/ISOPE18/All-ISOPE18/ISOPE-I-18-108/20128 (accessed on 22 December 2021).

2. Ran, Z.; Kim, M.H.; Zheng, W. Coupled Dynamic Analysis of a Moored Spar in Random Waves and Currents (Time-Domain Versus Frequency-Domain Analysis). ASME J. Offshore Mech. Arct. Eng. 1999, 121, 194-200. [CrossRef]

3. Amaechi, C.V.; Wang, F.; Hou, X.; Ye, J. Strength of submarine hoses in Chinese-lantern configuration from hydrodynamic loads on CALM buoy. Ocean Eng. 2019, 171, 429-442. [CrossRef]

4. Amaechi, C.V.; Wang, F.; Ye, J. Numerical assessment on the dynamic behaviour of submarine hoses attached to CALM buoy configured as lazy-S under water waves. J. Mar. Sci. Eng. 2021, 9, 1130. [CrossRef]

5. Amaechi, C.V.; Wang, F.; Ye, J. Numerical studies on CALM buoy motion responses, and the effect of buoy geometry cum skirt dimensions with its hydrodynamic waves-current interactions. Ocean Eng. 2022, 244, 110378. [CrossRef]

6. Edward, C.; Dev, A.K. Assessment of CALM buoys motion response and dominant OPB/IPB inducing parameters on fatigue failure of Offshore Mooring chains. In Practical Design of Ships and Other Floating Structures; PRADS 2019. Lecture Notes in Civil Engineering; Okada, T., Suzuki, K., Kawamura, Y., Eds.; Springer: Singapore, 2021; Volume 64. [CrossRef]

7. Shoup, G.J.; Mueller, R.A. Failure Analysis of a Calm Buoy Anchor Chain System. In Proceedings of the Offshore Technology Conference, Houston, TX, USA, 7-9 May 1984. [CrossRef]

8. Yang, C.; Kang, Z. Assessment of Fatigue Damage Initiation in FPSO's Oil Offloading Line in West Africa. In Proceedings of the 28th International Ocean and Polar Engineering Conference, Sapporo, Japan, 10-15 June 2018. Available online: https: / / onepetro.org/ISOPEIOPEC/proceedings-abstract/ISOPE18/All-ISOPE18/ISOPE-I-18-109/20158 (accessed on 2 May 2021).

9. Jean, P.; Goessens, K.; Hostis, D. Failure of chains by bending on Deepwater mooring systems. OTC-17238-MS. In Proceedings of the Offshore Technology Conference, Houston, TX, USA, 2-5 May 2005. [CrossRef]

10. Hasanvand, E.; Edalat, P. Sensitivity analysis of the dynamic response of CALM oil terminal, in the Persian Gulf region under different operation parameters. Int. J. Mar. Eng. 2020, 16, 73-84. [CrossRef]

11. Hasanvand, E.; Edalat, P. A comparison of the dynamic response of a product transfer system in CALM and SALM oil terminals in operational and non-operational modes in the Persian Gulf region. Int. J. Coast. Offshore Eng. 2021, 5, 1-14. Available online: http:/ /ijcoe.org/article-1-232-en.html (accessed on 22 December 2021).

12. Amaechi, C.V.; Wang, F.; Ye, J. Investigation on hydrodynamic characteristics, wave-current interaction, and sensitivity analysis of submarine hoses attached to a CALM buoy. J. Mar. Sci. Eng. 2022, 10, 120. [CrossRef]

13. Brady, I.; Williams, S.; Golby, P. A study of the forces acting on hoses at a monobuoy due to environmental conditions. In Proceedings of the Offshore Technology Conference, Houston, TX, USA, 5-7 May 1974; pp. 1-10. [CrossRef]

14. Amaechi, C.V.; Chesterton, C.; Butler, H.O.; Wang, F.; Ye, J. An overview on bonded marine hoses for sustainable fluid transfer and (un)loading operations via floating offshore structures (FOS). J. Mar. Sci. Eng. 2021, 9, 1236. [CrossRef]

15. Amaechi, C.V.; Chesterton, C.; Butler, H.O.; Wang, F.; Ye, J. Review on the design and mechanics of bonded marine hoses for Catenary Anchor Leg Mooring (CALM) buoys. Ocean Eng. 2021, 242, 110062. [CrossRef]

16. Amaechi, C.V.; Wang, F.; Ye, J. Mathematical modelling of bonded marine hoses for single point mooring (SPM) systems, with Catenary Anchor Leg Mooring (CALM) buoy application: A review. J. Mar. Sci. Eng. 2021, 9, 1179. [CrossRef]

17. Amaechi, C.V.; Wang, F.; Ja'e, I.A.; Aboshio, A.; Odijie, A.C.; Ye, J. A literature review on the technologies of bonded hoses for marine application. Ships Offsh. Struct. 2022, in press. [CrossRef]

18. Lassen, T.; Lem, A.I.; Imingen, G. Load response and finite element modelling of bonded offshore loading hoses. In Proceedings of the ASME 2014 33rd International Conference on Ocean, Offshore and Arctic Engineering, San Francisco, CA, USA, 8-13 June 2014; ASME: New York, NY, USA, 2014. Paper OMAE2014-23545. V06AT04A034. [CrossRef]

19. Amaechi, C.V.; Chesterton, C.; Butler, H.O.; Odijie, C.A.; Gu, Z.; Wang, F.; Hou, X.; Ye, J. Finite element modelling on the mechanical behaviour of Marine Bonded Composite Hose (MBCH) under burst and collapse. J. Mar. Sci. Eng. 2022, $10,151$. [CrossRef]

20. Amaechi, C.V.; Ye, J. A review of state-of-the-art and meta-science analysis on composite risers for deep seas. Ocean Eng. 2022, in press. 
21. Amaechi, C.V.; Gillet, N.; Hou, X.; Ye, J. Composite risers for deep waters using a numerical modelling approach. Compos. Struct. 2019, 210, 486-499. [CrossRef]

22. Amaechi, C.V.; Gillett, N.; Odijie, A.C.; Wang, F.; Hou, X.; Ye, J. Local and global design of composite risers on truss SPAR platform in deep waters. In Proceedings of the 5th International Conference on Mechanics of Composites. Instituto Superior de Tecnico, Lisbon, Portugal, 1-4 July 2019; no. 20005. pp. 1-3. Available online: https://eprints.lancs.ac.uk/id/eprint/136431/4 /Local_and_Global_analysis_of_Composite_Risers_MechComp2019_Conference_Victor.pdf (accessed on 22 December 2021).

23. Ryu, S.; Duggal, A.S.; Heyl, C.N.; Liu, Y. Prediction of Deepwater Oil Offloading Buoy Response and Experimental Validation Int. J. Offshore Polar Eng. 2006, 16, 290-296. Available online: https://www.sofec.com/wp-content/uploads/white_papers/2006 -ISOPE-Prediction-of-DW-Oil-Offloading-Buoy-Response.pdf (accessed on 22 December 2021).

24. Duggal, A.S.; Ryu, S. The Dynamics of Deepwater Offloading Buoys. WIT Trans. Built Environ. 2005, 84, 269-278. Available online: https://www.witpress.com/Secure/elibrary/papers/FSI05/FSI05026FU.pdf (accessed on 22 December 2021).

25. Salem, A.G.; Ryu, S.; Duggal, A.S.; Datla, R.V. Linearization of Quadratic Drag to Estimate CALM Buoy Pitch Motion in Frequency-Domain and Experimental Validation. ASME J. Offshore Mech. Arct. Eng. 2012, 134, 011305. [CrossRef]

26. Amaechi, C.V. Novel Design, Hydrodynamics and Mechanics of Marine Hoses in Oil/Gas Applications. Ph.D. Thesis, Lancaster University, Engineering Department, Lancaster, UK, 2021.

27. Amaechi, C.V.; Wang, F.; Ye, J. Experimental study on motion characterization of CALM buoy hose system under water waves. J. Mar. Sci. Eng. 2022, 10, 204. [CrossRef]

28. Lee, D.H.; Choi, H.S. A nonlinear stability analysis of tandem offloading system. In Proceedings of the 24th Symposium on Naval Hydrodynamics, Fukuoka, Japan, 8-12 July 2002; pp. 348-359. Available online: https://www.nap.edu/read/10834/chapter/25 (accessed on 22 December 2021).

29. Lee, D.H.; Choi, H.S. A stability analysis of tandem offloading systems at sea. J. Mar. Sci. Technol. 2005, 10, 53-60. [CrossRef]

30. Esmailzadeh, E.; Goodarzi, A. Stability analysis of a CALM floating offshore structure. Int. J. Non-Linear Mech. 2001, 36, 917-926. [CrossRef]

31. Cho, S.K.; Sung, H.G.; Hong, S.Y.; Kim, Y.H. Study of the Stability of Turret moored Floating Body. In Proceedings of the 13th International Ship Stability Workshop, Brest, France, 23-26 September 2013. Available online: http://www.shipstab.org/files/ Proceedings/ISSW /ISSW_2013_Brest_Brittany_France/07_Session_Special_problems/ISSW_2013_Cho_et_al.pdf (accessed on 22 December 2021).

32. Huang, H.; Chen, H.-C. Coupled CFD-FEM simulation for the wave-induced motion of a CALM buoy with waves modeled by a level-set approach. Appl. Ocean Res. 2021, 110, 102584. [CrossRef]

33. Gu, H.; Chen, H.-C.; Zhao, L. Coupled Mooring Analysis of a CALM Buoy by a CFD Approach. In Proceedings of the 27th International Ocean and Polar Engineering Conference, San Francisco, CA, USA, 25-30 June 2017; Paper no. ISOPE-I-17-223. pp. 646-653. Available online: https://www.researchgate.net/publication/320044188_Coupled_Mooring_Analysis_of_a_CALM_Buoy_by_ a_CFD_Approach (accessed on 22 December 2021).

34. Gu, H.; Chen, H.-C.; Zhao, L. Coupled CFD-FEM simulation of hydrodynamic responses of a CALM buoy. Ocean Syst. Eng. 2019, 9, 21-42. [CrossRef]

35. Gu, H. Coupled Mooring Analysis of a CALM Buoy by a CFD Approach. Master's Thesis, Texas A \& M University, College Station, TX, USA, 2016. Available online: https://hdl.handle.net/1969.1/159024 (accessed on 22 December 2021).

36. Toxopeus, S.; Sadat-Hosseini, H.; Visonneau, M.; Guilmineau, E.; Yen, T.G.; Lin, W.-M.; Grigoropoulos, G.; Stern, F. CFD, potential flow and system-based simulations of fully appended free running 5415M in calm water and waves. Int. Shipbuild. Prog. 2018, 65, 227-256. [CrossRef]

37. Bandringa, H.; Jaouën, F.; Helder, J.; Bunnik, T. On the Validity of CFD for Simulating a Shallow Water CALM Buoy in Extreme Waves. In Offshore Technology, Proceedings of the ASME 2021 40th International Conference on Ocean, Offshore and Arctic Engineering, Virtual, 21-30 June 2021; ASME: New York, NY, USA, 2021; Volume 1, V001T01A037. [CrossRef]

38. Woodburn, P.; Gallagher, P.; Naciri, M.; Borleteau, J. Coupled CFD Simulation of the Response of a Calm Buoy in Waves. In Proceedings of the ASME 2005 24th International Conference on Offshore Mechanics and Arctic Engineering, Halkidiki, Greece, 12-17 June 2005; ASME: New York, NY, USA, 2005; Volume 3, pp. 793-803. [CrossRef]

39. Woodburn, P.; Gallagher, P.; Ferrant, P.; Borleteau, J.P. EXPRO-CFD: Development and Validation of CFD Based Co-simulation of Spar/CALM Buoy Fluid Structure Interaction. In Proceedings of the Thirteenth International Offshore and Polar Engineering Conference, Honolulu, HI, USA, 25-30 May 2003. Available online: https:/ / onepetro.org/ISOPEIOPEC/proceedings-abstract/ ISOPE03/All-ISOPE03/ISOPE-I-03-225/8589 (accessed on 22 December 2021).

40. Bunnik, T.H.J.; de Boer, G.; Cozijn, J.L.; van der Cammen, J.; van Haaften, E.; ter Brake, E. Coupled Mooring Analysis and Large Scale Model Tests on a Deepwater CALM Buoy in Mild Wave Conditions. In Proceedings of the ASME 2002 21st International Conference on Offshore Mechanics and Arctic Engineering, Oslo, Norway, 23-28 June 2002; ASME: New York, NY, USA, 2002; Volume 1, pp. 65-76. [CrossRef]

41. Williams, A.N.; McDougal, W.G. Experimental Validation of a New Shallow Water CALM Buoy Design. In Offshore Technology, Proceedings of the ASME 2013 32nd International Conference on Ocean, Offshore and Arctic Engineering, Nantes, France, 9-14 June 2013; ASME: New York, NY, USA, 2013; Volume 1, V001T01A070. [CrossRef] 
42. Ricbourg, C.; Berhault, C.; Camhi, A.; Lécuyer, B.; Marcer, R. Numerical and Experimental Investigations on Deepwater CALM Buoys Hydrodynamics Loads. In Proceedings of the Offshore Technology Conference, Houston, TX, USA, 1-4 May 2006. [CrossRef]

43. Thilleul, O.; Drouet, A.; Andrillon, Y.; Guilcher, P.-M.; Jacquin, E.; Barcarolo, D.; Berry, L.; Ledoux, A.; Guillerm, P.-E.; Le Touzé, D.; et al. CFD tools and adapted methodologies for Marine and Offshore Engineering projects. In Proceedings of the Offshore Technology Conference, Houston, TX, USA, 4-7 May 2015. [CrossRef]

44. Le Cunff, C.; Ryu, S.; Duggal, A.; Ricbourg, C.; Heurtier, J.-M.; Heyl, C.; Liu, Y.; Beauclair, O. Derivation of CALM Buoy Coupled Motion RAOs In Frequency Domain and Experimental Validation. In Proceedings of the Seventeenth International Offshore and Polar Engineering Conference, Lisbon, Portugal, 1-6 July 2007; Paper Number: ISOPE-I-07-402. Available online: https:/ / www.sofec.com/wp-content/uploads/white_papers/2007-ISOPE-Derivation-of-CALM-Buoy-Coupled-MotionRAOs-in-Frequency-Domain.pdf (accessed on 22 December 2021).

45. Monroy, C.; Ducrozet, G.; Bonnefoy, F.; Babarit, A.; Gentaz, L.; Ferrant, P. RANS Simulations of a CALM Buoy in Regular and Irregular Seas Using the SWENSE Method. In Proceedings of the Twentieth International Offshore and Polar Engineering Conference, Beijing, China, 20-25 June 2010. Available online: https://www.researchgate.net/publication/254509441_RANS_ Simulations_of_a_Calm_Buoy_in_Regular_and_Irregular_Seas_using_the_SWENSE_Method (accessed on 17 December 2021).

46. Shah, Z.; Sheikholeslami, M.; Kumam, P.; Shutaywi, M.; Thounthong, P. CFD Simulation of Water-Based Hybrid Nanofluid Inside a Porous Enclosure Employing Lorentz Forces. IEEE Access 2019, 7, 177177-177186. [CrossRef]

47. Shah, Z.; Dawar, A.; Islam, S.; Alshehri, A.; Alrabaiah, H. A comparative analysis of MHD Casson and Maxwell flows past a stretching sheet with mixed convection and chemical reaction. Waves Random Complex Media 2021, 31, 1-16. [CrossRef]

48. Sagrilo, L.V.S.; Siqueira, M.Q.; Ellwanger, G.B.; Lima, E.C.P.; Ferreira, M.D.A.S.; Mourelle, M.M. A coupled approach for dynamic analysis of CALM systems. Appl. Ocean Res. 2002, 24, 47-58. [CrossRef]

49. SOFEC. SOFEC-Proven Technology Reliable Quality; SOFEC Inc.: Houston, TX, USA; pp. 1-20. Available online: https://www. sofec.com/wp-content/uploads/2020/02/SOFEC-Corporate-Brochure.pdf (accessed on 17 December 2021).

50. Cozijn, J.L.; Bunnik, T.H.J. Coupled Mooring Analysis for a Deep Water CALM Buoy. In Proceedings of the ASME 2004 23rd International Conference on Offshore Mechanics and Arctic Engineering, Parts A and B, pp. 1-11. Vancouver, BC, Canada, 20-25 June 2004; ASME: New York, NY, USA, 2004; Volume 1, pp. 663-673. [CrossRef]

51. Cozijn, H.; Uittenbogaard, R.; Brake, E.T. Heave, Roll and Pitch Damping of a Deepwater CALM Buoy with a Skirt. In Proceedings of the Fifteenth International Offshore and Polar Engineering Conference, Seoul, Korea, 19-24 June 2005; ISOPE-I-05-296. Available online: https://www.researchgate.net/publication/267364857_Heave_Roll_and_Pitch_Damping_of_a_Deepwater_ CALM_Buoy_with_a_Skirt (accessed on 17 December 2021).

52. Keulegan, G.H.; Carpenter, L.H. Forces on Cylinders and Plates in an Oscillating Fluid. J. Res. Natl. Bur. Stand. 1958, 60, 423-440. Available online: https://nvlpubs.nist.gov/nistpubs/jres/60/jresv60n5p423_A1b.pdf (accessed on 17 December 2021). [CrossRef]

53. Sarpkaya, T.; O’Keefe, J. Oscillating Flow about Two- and Three Dimensional Bilge Keels. J. Offshore Mech. Arct. Eng. 1996, 118, 1-6. [CrossRef]

54. Boccotti, P. Wave Mechanics for Ocean Engineering, 1st ed.; (Elsevier Oceanography Series); Elsevier Science Publishers: London, UK, 2000; Volume 64.

55. Boccotti, P. Wave Mechanics and Wave Loads on Marine Structures, 1st ed.; Elsevier Science Publishers, Imprint of ButterworthHeinemann Inc.: Woburn, MS, USA, 2014.

56. Dean, R.G.; Dalrymple, R.A. Water Wave Mechanics for Engineers and Scientists; (Advanced Series on Ocean Engineering); World Scientific Publishers: Toh Tuck Link, Singapore, 1991; Volume 2. [CrossRef]

57. McCormick, M.E. Ocean Engineering Mechanics: With Applications; Cambridge University Press: Cambridge, UK, 2010.

58. Eriksson, M.; Isberg, J.; Leijon, M. Theory and Experiment on an Elastically Moored Cylindrical Buoy. IEEE J. Ocean Eng. 2006, 31, 959-963. [CrossRef]

59. Jiang, D.; Zhang, J.; Ma, L.; Chen, H. Effect of heave plate on wave piercing buoy. In Proceedings of the 2016 Automotive, Mechanical and Electrical Engineering, Hong Kong, China, 9-11 December 2016; Taylor \& Francis Group: London, UK, 2017; pp. 367-370. [CrossRef]

60. Newman, J.N. Marine Hydrodynamics; 1999 Repri; MIT Press: London, UK, 1977.

61. Brebbia, C.A.; Walker, S. Dynamic Analysis of Offshore Structures, 1st ed.; Newnes-Butterworth \& Co. Publishers Ltd.: London, UK, 1979.

62. Paik, J.K.; Thayamballi, A.K. Ship-Shaped Offshore Installations: Design, Building, and Operation, 1st ed.; Cambridge University Press: Cambridge, UK, 2007.

63. Bishop, R.E.D.; Price, W.G. Hydroelasticity of Ships; Cambridge University Press: New York, NY, USA, 2005.

64. Rawson, K.J.; Tupper, E.C. Basic Ship Theory. Volume 1: Hydrostatics and Strength, 5th ed.; Butterworth-Heinemann: Oxford, UK, 2001.

65. Rawson, K.J.; Tupper, E.C. Basic Ship Theory. Volume 2: Ship Dynamics and Design, 5th ed.; Butterworth-Heinemann: Oxford, UK, 2001.

66. Zikanov, O. Essential Computational Fluid Dynamics; John Wiley \& Sons Inc., Wiley India Pvt. Ltd.: New Delhi, India, 2010.

67. Sarpkaya, T. Wave Forces on Offshore Structures, 1st ed.; Cambridge University Press: New York, NY, USA, 2014. 
68. Morison, J.R.; Johnson, J.W.; Schaaf, S.A. The Force Exerted by Surface Waves on Piles. J. Pet. Technol. 1950, 2, 149-154. [CrossRef]

69. Zhang, S.; Chen, C.; Zhang, Q.; Zhang, D.; Zhang, F. Wave Loads Computation for Offshore Floating Hose Based on Partially Immersed Cylinder Model of Improved Morison Formula. Open Pet. Eng. J. 2015, 8, 130-137. [CrossRef]

70. ANSYS. ANSYS Fluent Theory Guide; Release 15.0; ANSYS Inc.: Canonsburg, PA, USA, 2013. Available online: https://www. academia.edu/38091499/ANSYS_Fluent_Theory_Guide (accessed on 24 April 2021).

71. ANSYS. ANSYS Fluent User's Guide; Release 15.0; ANSYS Inc.: Canonsburg, PA, USA, 2013. Available online: http://www.pmt. usp.br/academic/martoran/notasmodelosgrad/ANSYS\%20Fluent\%20Users\%20Guide.pdf (accessed on 24 April 2021).

72. ANSYS. ANSYS Fluent Tutorial Guide; Release 14.0; ANSYS Inc.: Canonsburg, PA, USA, 2011. Available online: http://www. ansys.fem.ir/ansys_fluent_tutorial.pdf (accessed on 24 April 2021).

73. ANSYS. ANSYS Fluent Tutorial Guide; Release 18.0; ANSYS Inc.: Canonsburg, PA, USA, 2017. Available online: http:/ / users.abo. fi/rzevenho/ansys\%20fluent \%2018\%20tutorial\%20guide.pdf (accessed on 24 April 2021).

74. ANSYS. ANSYS Meshing User's Guide; Release 18.2; ANSYS Inc.: Canonsburg, PA, USA, 2017.

75. An, S.; Faltinsen, O.M. An experimental and numerical study of heave added mass and damping of horizontally submerged and perforated rectangular plates. J. Fluids Struct. 2013, 39, 87-101. [CrossRef]

76. Prislin, I.; Blevins, R.D.; Halkyard, J.E. Viscous Damping and Added Mass of Solid Square Plates. In Proceedings of the 7th Offshore Mechanics and Arctic Engineering Conference, Lisbon, Portugal, 5-6 July 1998. Paper Number: OMAE98-0316.

77. Martin, M. Rolling Damping due to Bilge Keels; Report prepared by the Iowa University Institute of Hydraulic Research for the Office of Naval Research under Contract No. 1611 (01); Iowa University Institute of Hydraulic Research: Iowa City, IA, USA, 1958. Available online: http:/ / resolver.tudelft.nl/uuid:cecb6d99-98b6-4d23-9cb8-d2e83714df59 (accessed on 17 December 2021).

78. Huang, Z.J.; Santala, M.J.; Wang, H.; Yung, T.W.; Kan, W.; Sandstrom, R.E. Component Approach for Confident Predictions of Deepwater CALM Buoy Coupled Motions: Part 1-Philosophy. In Proceedings of the ASME 2005 24th International Conference on Offshore Mechanics and Arctic Engineering, 24th International Conference on Offshore Mechanics and Arctic Engineering: Volume 1, Parts A and B, Halkidiki, Greece, 12-17 June 2005; ASME: New York, NY, USA, 2005; pp. 349-356. [CrossRef]

79. Santala, M.J.; Huang, Z.J.; Wang, H.; Yung, T.W.; Kan, W.; Sandstrom, R.E. Component Approach for Confident Predications of Deepwater CALM Buoy Coupled Motions: Part 2-Analytical Implementation. In Proceedings of the ASME 2005 24th International Conference on Offshore Mechanics and Arctic Engineering, 24th International Conference on Offshore Mechanics and Arctic Engineering: Volume 1, Parts A and B, Halkidiki, Greece, 12-17 June 2005; ASME: New York, NY, USA, 2005; pp. 367-375. [CrossRef]

80. Amaechi, C.V.; Wang, F.; Ye, J. Understanding the fluid-structure interaction from wave diffraction forces on CALM buoys: Numerical and analytical solutions. Ships Offsh. Struct. 2022, in press. [CrossRef]

81. Palm, J.; Eskilsson, C.; Paredes, G.M.; Bergdahl, L. Coupled mooring analysis for floating wave energy converters using CFD: Formulation and validation. Int. J. Mar. Energy 2016, 16, 83-99. [CrossRef] 